\title{
Singing sands, booming dune sands, and the stick-slip effect
}

\section{A. J. Patitsas}

Laurentian University, Department of Physics and Astronomy, Sudbury, ON, Canada, P3E2C6

e-mail: tpatitsas@sympatico.ca or tpatitsas@laurentian.ca

Revised edition, December 14, 2011.

Abstract: The origin of the acoustic and seismic emissions from impacted singing grains and from avalanching dune sand grains is sought in modes of vibration in discreet grain columns. It is postulated that when the grains in a column are pressed together, elastic shear bands are formed at the contact areas with distinct elastic properties. The central part of such contact shear bands, where the stress level is maximum, is partially fluidized, resulting in reduced elastic moduli. In a given column, the elastic moduli would assume the lowest values just below the impacting pestle and higher values further below. The assembly of all grain columns below the pestle forms the slip shear band. The transfer of energy from the pestle to the modes of vibration in such columns is effected by the stick-slip effect. The intense collective vibration of all columns in the slip shear band results in the familiar musical sound. The concept of grain flowability is used to justify the disparity between the acoustic emissions from impacted singing grains and from avalanching dune sand grains. The concept of grain columns is assumed to apply in the freely avalanching booming sand band, but with larger length to justify the lower frequencies. This approach predicts frequency spectra comprising a low frequency content and a dominant frequency with its harmonics in agreement with the experimental evidence. Additionally, it can account for the low frequency vibration evoked when booming sand flows through a funnel. It is argued that sand grains do not sing or boom since the stick-slip effect in not applicable in the contact shear bands.

PACS: 43.20.Ks, 89.75.Fb, 45.70.Mg, 43.40.Le 


\section{Introduction}

The mechanism responsible for the seismic and acoustic emissions, when a bed of singing sand or silica gel grains is impacted by a pestle, was the subject of a recent paper by Patitsas [1]. The mechanism was sought in shear modes of vibration in a well defined slip channel (shear band) comprising several grain layers ahead of the impacting pestle. It was argued that due to some not yet understood physico-chemical effect on the grain surface, a bed of singing (musical) grains is characterized by a relatively high level of rigidity. Thus, the grains just ahead of the pestle are subjected to a relatively high stress level resulting in the partial fluidization of the tips of the grain asperities and any grain coating at the contact areas, resulting in turn in drastically reduced elastic moduli in the slip channel.

However, the assumption in [1] that the length of the slip channel, in directions nearly normal to the direction of the pestle motion, is also well defined needs reconsideration. Such an assumption was necessary in order to view the slip channel as a cavity with well defined walls, with well defined shear standing wave patterns and with well defined eigenfrequencies. It is highly likely that the walls at the channel ends are not well defined and that there are propagating (traveling) waves along the slip channel as opposed to standing wave patterns. Thus, the determination of the frequencies of the seismic and acoustic emissions remains an open question. Whereas, it could be argued that the boundary of the slip channel adjacent to the pestle is well defined, i.e., the height of the bumps is small compared with the wavelength of any waves present, it is difficult to argue that this is also the case at the lower boundary. A more gradual transition from very low elastic moduli below the pestle to nominal values in the grain bed, well below the pestle, is a more realistic assumption. Effectively, the stress

level generated by the pestle decreases with depth resulting in lower fluidization at the grain contact areas. Furthermore, it is unreasonable to expect that continuum mechanics alone can provide a satisfactory solution when the width of the channel is only about ten times the particle (grain) size.

Similarly, there are serious questions that need be considered regarding the recent approaches in accounting for the seismic and acoustic emissions from avalanching dune sands. Four such approaches have been published over the past seven years: In the mainly experimental report by Andreotti [2], it is shown that during a booming dune avalanche, there are elastic waves propagating along the dune surface extending several $\mathrm{cm}$ below the surface. It is 
then argued that the grains would oscillate according to the particle displacement dictated by such waves. Furthermore, such waves would synchronize the grain-grain collisions and would become excited by such collisions. It is known that when grains of any kind are induced to avalanche down an inclined plane, the average time required by one grain to overtake another is given by the expression, $\left.T_{c}=1 / 0.4 \sqrt{(} \bar{d} / g\right)$, where $\bar{d}$ is the average grain diameter and $g=9.8 \mathrm{~m} / \mathrm{s}^{2}$, Andreotti [2], MiDi [3]. Thus, the dominant frequency of the propagating waves and that of the seismic and acoustic emissions can be defined as $f_{d}=1 / T_{c}$. However, in the paper by Vriend at al. [4], it is reported that dune vibrations were detected even when there was no apparent avalanche in progress and moreover, during avalanches the dominant frequency $f_{d}$ was accompanied by several harmonics.

Subsequent reports by Bonneau et al. [5, 6] go to great lengths to elucidate the properties of waves propagating along a dune surface where the elastic moduli increase with sand depth. However, there is no clear identification of the modes corresponding to frequencies equal to multiples of $f_{d}$. Even in the latest report by Andreotti and Bonneau [7], the question of harmonics of $f_{d}$ is not addressed. In this latter report, it is assumed that a thin shear band is formed between the avalanching sand band and the static sand below, and that leads to the excitation of the surface waves. Whereas, such a shear band is nearly evident when a sand plate breaks off and begins to slide downhill, there is no such evidence after the plate breaks up and a free avalanche ensues. On page 253 in Bagnold [8], it is stated that the velocity of a grain layer at depth $\zeta$ decreases linearly with $\zeta$ until it is zero at some depth $H_{o}$.

Furthermore, by studying a video recording by The National Geographic Society, Survivors of the Skeleton Coast Park, Namibia, Africa (1993), it can be concluded that the avalanche front looses height gradually until it comes to rest when the height is about 2 or $3 \mathrm{~cm}$. By observing the tail end of the avalanches in YouTube presentations, it can be inferred that its thickness is considerably lower than in the front. One such presentation by the authors of the paper by Douady et al. [9], with the title: The Song of Dunes, can be reached from the link in the same paper. Additionally, when the gate on a wood frame on the slip face of a boomable dune is suddenly released, with sand height behind the gate up to $10 \mathrm{~cm}$, [9], it is difficult to imagine that the thickness of the avalanching band is the same in its entire length. Therefore, the argument in [5] that for booming to occur the avalanche thickness must exceed a certain threshold is rather tenuous. Effectively, it is argued that the wave frequency defined by the 
overtake time $T_{c}$ would be lower than the cutoff frequency of propagation in the avalanching sand band. The argument is more tenuous when applied to the case of a sand pile pushed by a blade in the study by Douady et al. [9], where the geometry is even more ill-defined.

When, about $0.5 \mathrm{~kg}$ of booming sand grains from Sand Mountain, Nevada, USA, were placed in a glass jar, $7 \mathrm{~cm}$ in diameter by $16 \mathrm{~cm}$ in length, and shaken horizontally along the jar axis, the dominant frequency of the acoustic emission was $280 \mathrm{~Hz}$ (Leach and Rubin [10]). It can be argued that there is grain layer rollover and a high stress level when the grain mass collides with the jar wall, as there is layer rollover and high stress level when the avalanche front collides with the static sand ahead. The dominant frequency, $f_{d}$, is about four times larger in the former than in the latter case. According to Nori et al. [11], $f_{d}$ is approximately $65 \mathrm{~Hz}$ in the latter case. According to Leach et al. [12], $f_{d}$ tends to decrease with increased grain mass in the jar. It appears that the mechanism responsible for the emissions from highly localized events could also be responsible for the emissions from large scale events such as the pushing of the sand mass by the hand or a blade and the sliding of sand plates on a dune surface.

In the report by Patitsas [1], the concept of a shear band (slip channel) several mm thick, under a sliding sand plate or under a freely avalanching sand band, was used to explain the relevant emissions as originating with shear modes of vibration in the channel with shear phase velocity about $1 \mathrm{~m} / \mathrm{s}$, such that $\lambda \approx$ twice the channel thickness. But, even if such a channel existed in the case of free avalanche, it would not be well defined at the lateral ends, as in the case of the slip channel under an impacting pestle. However, this approach could explain the harmonics of $f_{d}$.

In the experimental report in [9], it is also recognized that the frequency, $f_{d}$, is defined by the overtake time, $T_{c}$, but the synchronization of the collisions is effected by some sort of coupling between adjacent grain layers due to some wave that propagates up-down between the static sand and the surface of the avalanching band. There is no attempt to account for overtone frequencies, but such an approach would lead to overtones in the sequence of $3 f_{d}, 5 f_{d}$ etc. However, the notion of up-down motion of grain layers is in agreement with the notion of up-down grain column oscillations proposed in this study.

In the mainly experimental report by Vriend et al. [4], the booming emission is sought in compression wave propagation along a surficial grain layer about $2 \mathrm{~m}$ in depth. The frequency 
is defined by the condition that for specific phase velocities in the substrate, the grain layer and in the air, there is total reflection at the boundaries. This approach was criticized by Andreotti et al. [13] especially regarding the assumption that the phase velocity does not increase with depth. Furthermore, the mathematical model used in [4] could not account for the low frequency content present in the free avalanche acoustic and seismic emissions. Additionally, it is highly unlikely that the energy generated by a few kg of freely avalanching sand grains into a hole dug on the face of a dune would be sufficient to excite a wave in such a large layer in thickness and length. The absence of boomability in certain dunes in a given area is not a strong indicator that the booming mechanism has to lie well beneath the dune surface, since on the surface all dunes appear to be the same. Equivalently, only certain sections of the Eastern and Northern shores of Lake Michigan USA, visited on August 2009, exhibited singability.

No explanation has been presented as to why booming dune sand would cease booming, when freely avalanching, but would continue to be musical when squeezed, Criswell et al. [14]. Furthermore, no arguments have been published as to the unexpected low propagation velocity of the synchronization wave on the booming dune surface [2], and more generally as to the unusually low propagation velocity of an elastic wave in a pile of ordinary sand, about $50 \mathrm{~m} / \mathrm{s}$, well below the values predicted by the Hertz-Mindlin theory, Bachrach et al. [15]. Finally, no satisfactory explanation exists as to why singing sands do not boom and booming sands do not sing, and as to why no acoustic emission is produced during an ordinary (silent) grain avalanche.

Before proceeding with the presentation of the present approach, it is deemed appropriate to attempt to elucidate the terminologies used in describing the sounds emitted by sheared granular media

- (a) Singing or squeaking sound refers to musical sound of short duration, up to $200 \mathrm{ms,}$ with frequencies in the range 250 to $2500 \mathrm{~Hz}$, emitted when beach sands or silica gel grains are impacted by an object or stepped on.

(b) Booming dune sound refers to a relatively low frequency, 60 to $100 \mathrm{~Hz}$, continuous humlike (drone-like) sound emitted when dune sand grains avalanche freely downhill.

(c) Continuous singing or squeaking sound refers to musical sound emitted when the bed of singing (squeaking) grains is sheared continuously by a vertical rod, immersed about $3 \mathrm{~cm}$, or 
by a smooth object pulled along the grain surface. The known frequency range is under 1000 $\mathrm{Hz}$.

(d) Pushed (squeezed) booming sand sound refers to a continuous musical sound emitted, roughly in the frequency range, 25 to $350 \mathrm{~Hz}$, when the sand mass is pushed continuously by the hand or a blade on the face of a dune, or by a blade in a confined geometry.

(e) Roar sound refers to loud sound emitted when the booming sand is pushed downhill in a heaped-up manner, Lewis [16]. The frequency range has not been recorded but it is likely at about $200 \mathrm{~Hz}$.

(f). Sand plate sound refers to the sound emitted when dune sand plates avalanche freely downhill. The frequency range has not been recorded but it is likely at about $200 \mathrm{~Hz}$.

\section{List of symbols used extensively}

$d \quad$ overall diameter of a grain

$R \quad \mathrm{R}=\mathrm{d} / 2$

$\bar{d}$ average diameter of grains in a column

$b \quad$ thickness of a contact shear band between two grains

$R_{b} \quad$ radius of a contact shear band

$c_{p} \quad$ compression phase velocity in a contact shear band

$c_{s} \quad$ shear phase velocity in a contact shear band

$\hat{x} \quad$ unit vector along the direction of grain-grain slide

$\hat{z} \quad$ unit vector along a grain column

$z \quad$ distance from the bottom of a grain column towards the pestle above

$\zeta$ sand depth on the face of a dune

$\xi_{x} \quad$ particle displacement along $\hat{x}$ inside the contact shear band

$\xi_{z} \quad$ particle displacement along $\hat{z}$ inside the contact shear band

$L \quad$ length of the contact shear band along $\hat{x}$ equal approximately to $2 R_{b}$

$\lambda_{x} \quad$ wavelength along a grain layer along the direction of slide

$\lambda_{z} \quad$ wavelength along a grain column

$N$ number of grains in a column

$V_{c} \quad$ compression propagation velocity in a grain column

$\lambda_{c} \quad$ wavelength of a mode of vibration along a column, same as $\lambda_{z}$ 
$\eta \quad$ correction factor in (9)

$M_{p} \quad$ equivalent pestle mass above a grain column

$f_{1} \quad$ frequency of the fundamental mode in a grain column

$f_{2}, . . f_{N} \quad$ overtone frequencies, on average, harmonics of $f_{1}$

$f_{p} \quad$ frequency of the pestle mode of vibration where the entire column acts like a sort spring

$T_{c} \quad$ time for one grain to overtake another $=1 / 0.4 \sqrt{(\bar{d} / 9.8)}$

$f_{c}$ grain-grain collision frequency in a surface grain avalanche $=1 / T_{c}$

$f_{d} \quad$ frequency at the center of the dominant experimental spectrum envelope

$H$ height of drop of a pestle on the grain bed surface

Brevort River sand: singing sand collected at the mouth of the Brevort River flowing into the North shore of Lake Michigan, USA, about $25 \mathrm{~km}$ west from the city of St. Ignace. Large plastic container: a $46 \times 28 \mathrm{~cm}$ by $10 \mathrm{~cm}$ in sand depth plastic container

\section{The grain column approach}

Figure 1 depicts an assumed grain configuration inside a slip channel where the five grain layers slide over one another along $\hat{x}$. For reasons to become clear later, the slip channel can also be referred to as, the vibration shear band or

the column shear band or even better, the slip shear band. Ultimately the source of all vibrations are the elastic shear bands at the grain contact areas. For the first column on the left hand side, they are labeled as: shear band \# 1 at the bottom to shear band \#6 at the top. It is understood that the lifetime of a given column is roughly equal to the average time required for a grain to overtake another, $T_{c}$, and that the lifetime of the five column configuration is about five times shorter and that the lifetimes would decrease with increased grain number, $N$, in the columns. However, the duration of the signals generated when a small steel sphere impacts a grain bed, Fig. 2 for example, is comparable to the time $T_{c} \approx 35 \mathrm{~ms}$ for relative slippage velocity, $1 \mathrm{~cm} / \mathrm{s}$, and grain diameter, $d=0.35 \mathrm{~mm}$. As the sphere descends into the grain bed, a given column, about 5 to $10 \mathrm{~mm}$ long, looses grains at the top and gains grains at the bottom. There is also grain exchange between columns, but, the collective vibration of the columns, outlined below, would tend to smooth out the frequency shifts in the course of time. 
These disk-like shear bands are characterized by thickness, $b$, radius, $R_{b}$, and by compression and shear moduli that result in the corresponding phase velocities, $c_{p}, c_{s}$. In the context of the analysis that follows, $b$ is assumed to be in the order of $300 \mathrm{~nm}$ for average grain dimeter, $\bar{d} \approx 0.3 \mathrm{~mm}$, higher values can be compensated by higher values of the elastic moduli. In the initial computations that follow, $c_{p}, c_{s}$, in the contact bands immediately below the pestle, assume values in the order of a few $\mathrm{m} / \mathrm{s}$, implying a soft physical composition of such contact bands.

Fig.1. An assumed grain column configuration in a slip shear band. The shaded areas correspond to the elastic contact shear bands with physical properties of their own when the grains are forced to slide past one another. They are characterized by compression and shear phase velocities, $c_{p}, c_{s}$ and particle displacements $\xi_{z}, \xi_{x}$.

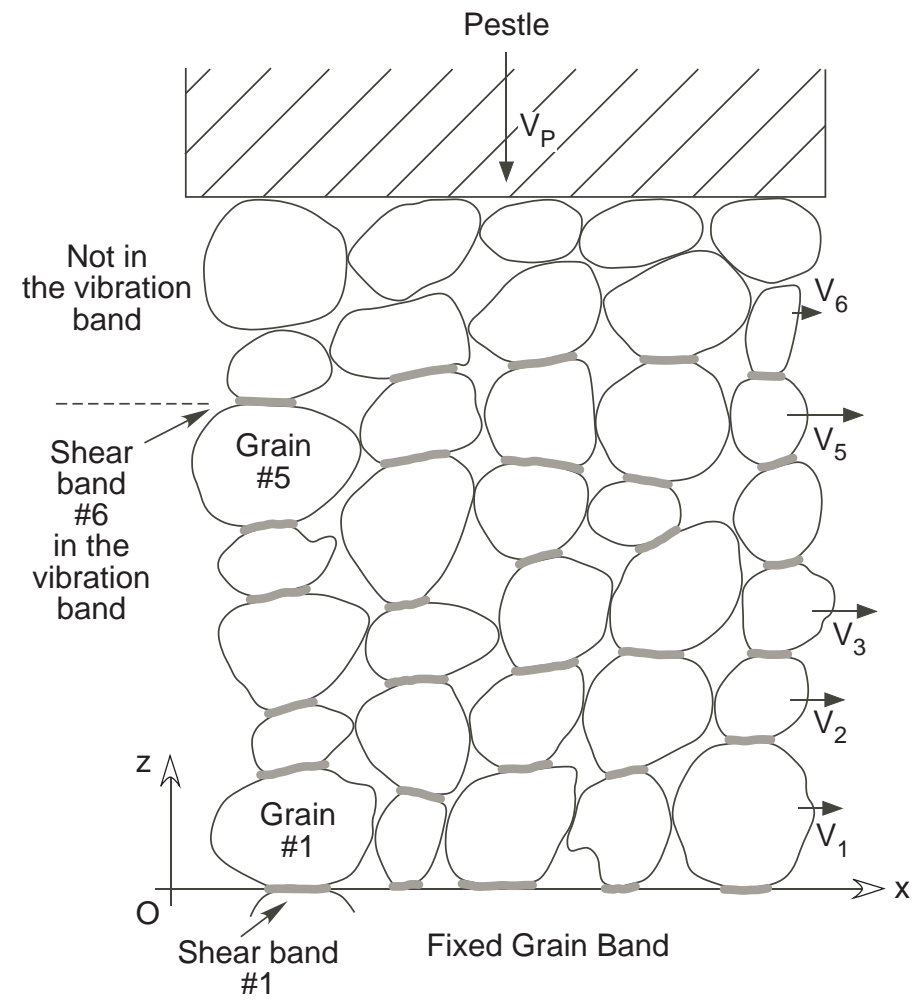

Inside the contact shear bands lie the tips of the surface asperities where transient temperatures can rise to several hundred $C^{o}$ in time intervals up to a few microseconds, Bhushan [17]. It is widely assumed that the space between the asperities is filled with some sort of coating that plays a central role in the production of the musical sound. Several references 
to this effect can be found in $[1,9]$. In the report by Lewis [16], the negative effect of moist conditions on the boomability of sand grains is stressed and in the report by Miwa et al. [18], it is suggested that certain beach sands lost their singability due to water pollutants resulting from nearby construction projects. In the report by Brunet et al. [19], it is shown that in a glass bead packing subjected to ultrasound waves, the dissipation increased by a factor of five when the beads were covered with silicon oil. However, the loss of musicality of polluted grains may not be as much due to viscous absorption as to changes in the friction coefficient that results in the non-applicability of the stick-slip effect [20].

In a recent report by Patitsas [20], it is demonstrated that the water layer on the epidermis of a finger rubbed on a glass surface acts as the interfacial band that facilitates slipping and also results in the decrease of the friction coefficient with relative velocity resulting in the stick-slip effect. Furthermore, it is argued that the shear modes of vibration responsible for the acoustic emission are to be found in the finger skin. However, there is no reason why the modes of vibration in the skin with thickness $b_{s} \approx 5 \mathrm{~mm}$, shear phase velocity $c_{s} \approx 10 \mathrm{~m} / \mathrm{s}$ and wavelength $\lambda \approx 2 b_{s}$ could not also exist in the interfacial band with $c_{s} \ll 10 \mathrm{~m} / \mathrm{s}$ and thickness $b \ll \lambda$. In this sense, the shear bands in Fig. 1 are assigned the roles of the interfacial band and of the site of the shear modes of vibration with $b \ll \lambda$. The attempt to write, $\lambda=2 b$ results in $c_{s} \approx 6 \times 10^{-4} \mathrm{~m} / \mathrm{s}$, for $b=\bar{d} / 1000$ and $f_{d}=1000 \mathrm{~Hz}$, where the average grain diameter is, $\bar{d}=0.3 \mathrm{~mm}$. Such unrealistic low value of $c_{s}$ leads to the conclusion that the shear modes of vibration in the contact shear bands must be characterized by the conditions, $b \ll \lambda$ and $L \ll \lambda$ along $\hat{z}$ and $\hat{x}$ respectively in Fig. 1 , where $L$ is the length of a given band along $\hat{x}$. It will be argued below that $\lambda$ is the wavelength of the wave motion along and normally to the grain column, i.e., such modes of vibration exist as part of macroscopic wave motion in the adjacent grain mass.

If the motion of the grains in a given column were along $\hat{z}$ only, the contact shear bands could be replaced by equivalent short weightless springs. It is a straightforward exercise to compute the eigenfrequencies and describe the corresponding modes of vibration for such a system. For $N$ blocks and $N+1$ springs, there are $N$ modes of vibration with frequencies, $f_{1}, f_{2} \ldots f_{N}$. For the mode with frequency $f_{1}$, all blocks oscillate in phase while for the mode with frequency $f_{N}$, neighboring blocks oscillate out of phase. The frequency $f_{1}$ tends to be rather insensitive to permutations of the blocks with different mass. 
It appears suitable at this stage to include a short paragraph from the study by Haff [21]. While the author was thinking of the booming dune emissions, the implications for the impacted grains are obvious. "Perhaps the mechanical analogue which most readily comes to mind is the slipstick phenomenon, a nonlinear mechanism by which a steady input of external energy is ultimately released and stored. This is certainly consistent with the oscillatory nature of the system and with its sensitivity to grain surface conditions and hence, presumably, to friction. To ascribe booming to a slipstick mechanism, however, is only to say the words; until we have a clear picture in mind of what the grains are actually doing, we do not really understand the origin of the booming sands".

\section{Modes of vibration in a given grain column}

In what follows, the origin, $O$ in Fig. 1, is assumed to coincide with the left side of shear band \# 1. The particle displacement, $\xi_{s}$, is written as, $\xi_{\mathbf{s}}=\nabla \times \mathbf{A}$, where $\mathbf{A}$ satisfies the vector wave equation with phase velocity $c_{s}$. A is chosen to lie along $\hat{y}$ resulting in, $A_{y}=\left[A_{1} \cos \alpha z+B_{1} \sin \alpha z\right]\left[A_{1}{ }^{\prime} \cos \beta x+B_{1}^{\prime} \sin \beta x\right] \mathrm{e}^{j \omega t}$ and this in turn results in, $\xi_{z}=\beta\left[A_{1} \cos \alpha z+\right.$ $\left.B_{1} \sin \alpha z\right]\left[-A_{1}^{\prime} \sin \beta x+B_{1}^{\prime} \cos \beta x\right]$ and, $\xi_{x}=\alpha\left[A_{1} \sin \alpha z-B_{1} \cos \alpha z\right]\left[A_{1}^{\prime} \cos \beta x+B_{1}^{\prime} \sin \beta x\right]$, where the factor $\mathrm{e}^{j \omega t}$ is understood to be included. The wave number, $k_{s}=\omega / c_{s}$, is given as, $k_{s}^{2}=\alpha^{2}+\beta^{2}$. The question arises as to the nature of the boundary conditions at the ends of a given shear band. If the ends are free, then, $\partial \xi_{x} / \partial x=0$ at $x=0$, resulting in $B_{1}^{\prime}=0$ and in, $\xi_{z}=\left[A_{1} \cos \alpha z+B_{1} \sin \alpha z\right] \beta \sin \beta x$ and, $\xi_{x}=\left[A_{1} \sin \alpha z-B_{1} \cos \alpha z\right] \alpha \cos \beta x$. The problem with the choice of free ends is that $\xi_{x}$ is greater than $\xi_{z}$ by several orders of magnitude since $\beta x \rightarrow 0$ for the low frequency modes and $\beta \ll \alpha$ since $b \ll L$. If the ends are fixed then,

$$
\xi_{z}=\left[A_{1} \cos \alpha z+B_{1} \sin \alpha z\right] \beta \cos \beta x
$$

and

$$
\xi_{x}=\left[A_{1} \sin \alpha z-B_{1} \cos \alpha z\right] \alpha \sin \beta x
$$

The latter choice appears reasonable when it is realized that the stress level decreases rapidly as $x$ approaches the ends of the band resulting in a solid-like state at the ends.

In shear band $\# 1$, the expression for the particle displacement along $\hat{z}$ simplifies to,

$$
\xi_{1 z}=\left[B_{1} \sin \alpha z\right] \beta \cos \beta x
$$


assuming that $\xi_{1 z}=0$ at $z=0$, and that in shear band $\# 2$ becomes,

$$
\xi_{2 z}=\left[A_{2} \cos \alpha z+B_{2} \sin \alpha z\right] \beta \cos \beta x
$$

In shear band \#3, the coefficient subscripts become 3 and so on for the rest of the bands.

The boundary condition on the top of shear band \# 1 at $z=b$ is,

$$
\int \sigma_{1 z z} d x d y+\int \sigma_{2 z z} d x d y=M_{1} \partial^{2} \xi_{1 z} / \partial t^{2}
$$

where $M_{1}$ is the mass of grain $\# 1$ and $\partial^{2} \xi_{1 z} / \partial t^{2}$ is evaluated at some point $x \rightarrow 0$. The normal stress per unit area along $\hat{z}$ is given as, $\sigma_{1 z z}=-\left(\lambda_{e}+2 \mu_{e}\right) \partial \xi_{1 z} / \partial z=-\rho c_{p}^{2} \partial \xi_{1 z} / \partial z$, while that along $-\hat{z}$ at the bottom of shear band $\# 2$ is given as, $\sigma_{2 z z}=\rho c_{p}^{2} \partial \xi_{2 z} / \partial z$. The mass density in the bands was assumed to be equal to that in the grains, i.e., that of quartz equal to $2650 \mathrm{~kg} / \mathrm{m}^{3}$. Equation (5) is repeated at the top of shear band \# 2 until the top of the last band $\# 6$, where the normal shear force $\sigma_{6 z z}$ acts on the equivalent pestle mass $M_{p}$, i.e.,

$$
\int \sigma_{6 z z} d x d y=M_{p} \partial^{2} \xi_{6 z} / \partial t^{2}
$$

The result of (5), with $A_{1}=0$, is the following working equation,

$$
\left[-S_{1} \rho c_{p}^{2} \alpha \cos (\alpha b)+M_{1} \omega^{2} \sin (\alpha b)\right] B_{1}-\left[S_{2} \rho c_{p}^{2} \alpha \sin \left(\alpha_{1}\right)\right] A_{2}+\left[S_{2} \rho c_{p}^{2} \alpha \cos \left(\alpha_{1}\right)\right] B_{2}=0
$$

where $S_{1}, S_{2}$ are the areas of bands \# 1 and 2 , and $\alpha_{1}=\alpha\left(b+d_{1}\right)$.

The grains are assumed to be perfectly rigid, so that $\xi_{1 z}(z=b)=\xi_{2 z}\left(z=b+d_{1}\right)$, resulting in the working equation,

$$
\sin (\alpha b) B_{1}-\cos \left(\alpha\left(b+d_{1}\right)\right) A_{2}-\sin \left(\alpha\left(b+d_{1}\right)\right) B_{2}=0
$$

where $d_{1}$ is the overall diameter of grain\#1. There are $2 N+1$ equations and $2 N+1$ coefficients, $B_{1}, A_{2}, B_{2}, \ldots A_{6}, B_{6}$ for $N=5$ grains as in Fig. 1. 


\section{Propagation velocity in a grain column}

It will be seen below that the present approach can account, fairly satisfactorily, for the observed frequency spectra. However, it ought to account, even to some vague degree, for the vaguely known seismic propagation velocity in a grain bed inside a bowl. To this end, an attempt is made here to derive an expression for the propagation velocity in a column (chain) of grains lying on a frictionless horizontal floor in order to emphasize the irrelevance of the force of gravity. The grains are assumed to be spherical and identical with diameter, $d$, mass, $m$, and the contact shear bands with thickness, $b$, are replaced by identical short springs of spring constant, $k$. If an equivalent Young's modulus for the thin contact bands is defined as, $Y_{b}=F /(\delta z / b)$, then, $Y_{b}=k b$. On page 305 in Symon [22], the problem of wave propagation in a string (chain) of point particles of mass $m$, interparticle distance, $h$, and string tension, $\tau$, is treated. A similar procedure can be used in this case by writing the force equation for the $n^{\text {th }}$ grain as, $m d^{2} u_{n} / d t^{2}=k\left(u_{n-1}-u_{n}\right)-k\left(u_{n}-u_{n-1}\right)$ where the displacement of the $n^{t h}$ grain is, $u_{n} \approx n d$, since $b \ll d$. Then, by introducing the mass per unit length, $\sigma=m / d$, the wave equation can be obtained, with phase velocity, $V_{c}=\sqrt{k d / \sigma}=\sqrt{(d / b)\left(Y_{b} / \sigma\right)}$. Furthermore, if the compression phase velocity in the contact bands is defined as, $c_{p}=1 / \eta \sqrt{\left(Y_{b}\right)\left(/ \sigma_{b}\right)}$, then, $V_{c} / c_{p}=\eta \sqrt{(d / b)\left(\sigma_{b} / \sigma\right)}$, where $\eta$ is an adjustment constant. It can be shown that, $\sigma_{b} / \sigma=3 / 2\left(R_{b} / R\right)^{2}$, where, $R_{b}, R$ are the radii of the contact bands and the grains respectively, with the assumption that the mass densities in the grains and the contact bands are the same. Thus,

$$
V_{c} / c_{p}=\eta \sqrt{3}\left(R_{b} / R\right) \sqrt{R / b}
$$

It is worthy of note that the equivalent spring constant, $k$, varies as $R_{b}^{2}$ and as $1 / b$ and thus,

$V_{c}$ varies as $R_{b} \sqrt{1 / b}$. It will be argued below that $R / R_{b}=22.5$ and that $R / b=500$, resulting in $V_{c} / c_{p}=\eta \sqrt{3}$

\section{Computations and implications}

The major difficulty with this study is that the only parameters that can be measured with certainty are the dominant frequency of the seismic and acoustic emissions, $f_{d}$, and at times harmonics of $f_{d}$. In the computations that follow, the contact band thickness and radius, $b, R_{b}$, respectively and the number of grains in a given column, $N$, are assigned reasonable 
values and the phase velocities, $c_{p}, c_{s}$, are allowed to vary. The eigenfrequencies, $f_{1}, f_{2}, . . f_{N}$, are then determined by looking for the zeros of the determinant of the coefficients, $B_{1}, A_{2}, .$. when $\omega=2 \pi f$ is varied, provided the wavenumber $\alpha$ can be specified in terms of $\omega$. Then, for a given eigenfrequency, the coefficients can be specified relative to the arbitrary value of $B_{1}=1$, and the nature of the corresponding mode of vibration can be examined. However, the value of the wavenumber $\beta$ must be specified before $\alpha$ can be specified from the relation, $\left(\omega / c_{s}\right)^{2}=\alpha^{2}+\beta^{2}$. It was argued above that for low frequency vibrations, $\lambda_{z} / b \gg 1$ and also $\lambda_{x} / L \gg 1$. But, $L / b \approx 100$ and thus it can be argued that $\lambda_{x} / \lambda_{z} \approx 100$, resulting in $\alpha / \beta \approx 100$, and $\alpha \approx \omega / c_{s}$. Thus, it is argued that the relation between $\alpha$ and $\beta$ for a standing wave pattern in a given contact shear band must also hold for a low frequency mode of vibration. The attempt to specify $\beta$ from the condition, $\xi_{x}=0$ at $x=L$ results in $\beta=\pi / L$ from (2). However, a finite value of $\beta$ results in a cutoff frequency that is not consistent with the experimental results that follow and also with the need for a low frequency corresponding to pestle vibration.

The question arises naturally as to the value of the number, $N$, of grains in a given grain column. Figures 8 and 9 in [1] depict the acoustic emissions when sand grains in a porcelain coffee cup, $9 \mathrm{~cm}$ in diameter, were impacted by a wood rod $25 \mathrm{~mm}$ in diameter. The sand depth before impact was about $10 \mathrm{~mm}$ and that after impact about 4 or $5 \mathrm{~mm}$, implying that $N \approx 15$.

Computations were commenced by choosing $N=12$ and average grain dimeter $\bar{d}=0.35 \mathrm{~mm}$ to correspond to that of the singing sand collected from the mouth of the Brevort River flowing into the north shore of Lake Michigan, USA, about $25 \mathrm{~km}$ west from the city of St. Ignace. The grain diameter, $d_{j}$, was varied randomly between 0.2 and $0.5 \mathrm{~mm}$ and the band thickness was assigned the value, $b=\bar{d} / 1000$. The circular contact areas, $S_{j}$ in (7), were evaluated by assuming the radii to be equal to the average diameter of the adjacent grains divided by 45. When two spheres of diameter, $d$, are squeezed together until the distance between their centers becomes $d-b$, then, the radius of the contact shear band is, $R_{b}=(1 / \sqrt{2}) \sqrt{b d}=d / 45$ for $b=d / 1000$. However, there is flexibility in this theoretical approach, i.e., if $R_{b}$ is twice as large and $b$ is four times as large, $V_{c}$ in (9) remains the same and so do the eigenfrequencies as pointed out below.

When a rod is hand-held and pushed or tapped into a sand bed, it is impossible to estimate 
the pestle mass $M_{p}$. However, when a small sphere is dropped on the sand bed, it is possible to evaluate approximately the effective value of $M_{p}$ on top of a given column. To this end, the sum of the cross-sectional areas of the columns below the sphere was assumed to be equal to $1 / 3$ the sphere cross-sectional area.

It is reasonable to assume that the degree of fluidization of the grain contact bands is highest immediately below the pestle and that it decreases gradually with depth until it is equal to that in the un-sheared static grains, assuming no wall effects. There would be a gradual tapering off near the bottom of the grain column, however, for reason of computation simplicity a linear decrease of the degree of fluidization with depth was assumed throughout the column. The value of the compression velocity in the contact band below the pestle was assigned the convenient value, $c_{p}(13)=1.0 \mathrm{~m} / \mathrm{s}$, and $c_{s}$ was assigned the same value as $c_{p}$ at this stage for lack of any information otherwise. Then, the value of $c_{p}(1)=12.0 \mathrm{~m} / \mathrm{s}$ resulted in $f_{1}=f_{d}=812 \mathrm{~Hz}$ corresponding to the dominant peak in Fig. 2. The sequence of the frequencies, $f_{p}, f_{1}, f_{2}, f_{3}, . . f_{12}$, was as follows: $30,812,1497,3091 \mathrm{~Hz}, .$. with corresponding $\alpha b=2 \pi(b / \lambda)$ values of: $0.000001,0.0003,0.0005,0.0011, \ldots$ For this particular grain size distribution, $f_{3}$ is excessively high.

When all grain diameters were set equal to $0.35 \mathrm{~mm}$, the frequencies, $f_{i}$, were, 36,831 , $1532,2284 \mathrm{~Hz}, \ldots$ All 12 modes conform with the condition, $b / \lambda \ll 1$. The values, $c_{p}(1)=12$ $\mathrm{m} / \mathrm{s}, c_{p}(13)=1 \mathrm{~m} / \mathrm{s}$, are not unique, i.e., practically the same frequencies, $f_{i}$, could be obtained with $c_{p}(13)=2 \mathrm{~m} / \mathrm{s}$ and with $c_{p}(1)$ somewhat lower than $12 \mathrm{~m} / \mathrm{s}$. It will be argued below in Section 11 that the elastic moduli tend to vanish near the grain bed surface where, $c_{p}=1 \mathrm{~m} / \mathrm{s}$ could not be ruled out. Furthermore, it will be argued at the end of this section that this approach can allow for considerably higher values $c_{p}(1), c_{p}(13)$, a few $\mathrm{cm}$ below the surface, when it is recognized that the effect of the pestle stress on the grains results not only in lower values for $c_{p}(j)$ but also in lower contact surface areas $S_{j}$ due to grain-grain relative slippage.

The lowest frequency, $f_{p}=30 \mathrm{~Hz}$, corresponds to the pestle vibration and it decreases rather strongly with increased pestle mass $M_{p}$ while the frequencies $f_{j}$ decrease very weakly with increased $M_{p}$. This is in agreement with the lack of significant sensitivity of the frequency, $f_{d}=$ $f_{1}$, on the length of the rod used to impact the grain bed or the manner by which the impaction is effected, by pushing or tapping the rod, for example. The frequencies corresponding to standing wave patterns in the contact shear bands are quite high, i.e., $\alpha b=\pi$ results in 
$f=1 / 3.5 \times 10^{6} \mathrm{~Hz}$ with $b=0.35 / 1000 \mathrm{~mm}$.

Variations in the values of $c_{s}, c_{p}$ revealed that the frequencies $f_{i}$ are proportional to the values of $c_{p}$ and nearly independent of the values of $c_{s}$. Only when $c_{s}(j)$ were reduced to $c_{p}(j) / 200$ there was an appreciable reduction in $f_{i}$, except for $f_{p}$, namely, $28 \mathrm{~Hz}(\alpha b=0.002)$, $701 \mathrm{~Hz}(0.06), 1487 \mathrm{~Hz}(0.15), 2220 \mathrm{~Hz}(0.19)$. It could be argued that when $c_{s}=c_{p}, \alpha=\omega / c_{s}$ is extremely small, resulting in extremely large wavelength $\lambda_{c}$ along the grain column compared with the contact band thickness, $b$. The lack of dependence of $f_{i}$ on $c_{s}$ can also be justified in the way the equations above were based on the particle displacement along $\hat{z}$ as opposed to $\hat{x}$ where the shear strain is present. The proportionality between the frequencies $f_{i}$ and $c_{p}(j)$ implies that $(9)$ represents adequately the compressive wave propagation in the grain column, since a given $f_{i}$, corresponding to a given standing wave pattern in the column, is proportional to $V_{c}$. The same argument can be repeated in justifying why the frequencies, $f_{1}, f_{2}, \ldots$ are reduced by the factor of $\sqrt{2}$ when the ratio $\bar{d} / b$ is reduced by the factor of 2.0 , why the same frequencies are increased by the factor 2 when the contact band radii are increased by the same factor, and why the same frequencies are reduced by the factor of 2.0 when the grain diameters are increased by the same factor.

The description of the corresponding modes was effected by computing the coefficients, $B_{1}, A_{2}, B_{2}, . . A_{13}, B_{13}$, and then the particle (grain) displacements, $\xi_{z}, \xi_{x}$, at the bottom middle and top of every contact band. It was verified that, when $c_{p}(j)$ are all constant, regardless of the number $N$ of grains in the column, the particle displacement, $\xi_{z}$, behaves like a half sine function, i.e., it peaks at the middle of the column and then dips to zero at the top of the column . Then, according to $(1,2), \xi_{x}$ has a node at the middle of the column. In simple terms, this is the fundamental mode of an elastic thin bar fixed at both ends. However, when $c_{p}(j)$ are assigned lower values as $j$ approaches $N+1, \xi_{z}$ attains maximum value somewhat above the middle of the column and thus, $\xi_{x}$ has a node correspondingly. Generally, $\xi_{x}$ is about ten times as large as $\xi_{z}$.

The relatively large particle displacement along $\hat{x}$ is consistent with the assumption that the stick-slip effect plays an important role towards the realization of the musical acoustic emission. In the case of the compression waves in the contact bands, $\xi_{z}>\xi_{x}$ and since $\xi_{z}$ is constrained to remain small, such waves cannot become excited. This question is raised also in [20]. From (8), it follows that $\xi_{z}$ is also the grain displacement along $\hat{z}$, and since $L \ll \lambda_{x}$, 
$\xi_{x}$ is a measure of the grain oscillation displacement along $\hat{x}$.

When the number of grains in a given column was increased from 12 to 24, with the same average diameter, $\bar{d}=0.35 \mathrm{~mm}$, and the same values for $c_{p}(j)$, the eigenfrequencies were reduced by the factor of 2.0 , since the propagation velocity in the column, $V_{c}$, remained the same but the wavelength was increased by the same factor. Furthermore, when the grains were permuted in several ways, $f_{1}$ remained in the range of, $800 \pm 80 \mathrm{~Hz}$, provided the smaller grains were not appreciably segregated from the larger grains. The spread about the central value of $f_{1}$ lies within the half width of the major frequency envelopes, Fig. 2, for example. A spread of about \pm 10 per cent was also observed around the frequency $f_{2}$. If all $c_{p}(j)$ were increased by a factor of 2.0 , then the values of $f_{i}$ would be the same as for $N=12$, but since $c_{p}(25)$ remains at $1.0 \mathrm{~m} / \mathrm{s}, c_{p}(1)$ has to be increased somewhat more than by the factor of 2.0, i.e., from 12.0 to $26.0 \mathrm{~m} / \mathrm{s}$, and for $N=48$, to $60.0 \mathrm{~m} / \mathrm{s}$. Thus, it can be concluded that generally a stiffer grain mass implies longer grain columns for the same frequency $f_{d}$.

It is now argued that for a given $N$, the propagation velocity, $V_{c}$, in a column of unsheared grains extending from the free surface to the depth, $H=N \bar{d}$, could be determined by assuming that $c_{p}(j)=c_{p}(1)$ for all $j$, and then by computing the dominant frequency $f_{1}$. Then with $\lambda_{c} \approx 4 H, V_{c}=f_{1} \lambda_{c}$. Computations revealed that $V_{c}(N=12) \approx 18.5 \mathrm{~m} / \mathrm{s}$, $V_{c}(N=24) \approx 43.3 \mathrm{~m} / \mathrm{s}$, and $V_{c}(N=48) \approx 101.6 \mathrm{~m} / \mathrm{s}$. Thus, every time $N$ is doubled, $V_{c}$ is increased by about the factor of 2.34 . For $N=12,24$ and 48 , the ratios $V_{c} / c_{p}(1)$ are 1.54, 1.66 and 1.69 respectively. Thus, the constant $\eta$ introduced in Section 4 is nearly equal to 1.0. It is reasonable to assume that the velocity, $V_{c}$, is a measure of the velocity of a compression wave propagating in an undisturbed grain bed.

It is reported in [4] that "body wave velocities" in the range 180 to $300 \mathrm{~m} / \mathrm{s}$ were measured well below the sand dune surface, and in Fig. 24.23 in Winterkorn and Fang [23], shear wave velocities as low as $150 \mathrm{~m} / \mathrm{s}$ are reported for dry round and angular-grained sands. Additionally, wave velocities as low as $50 \mathrm{~m} / \mathrm{s}$ in ordinary sand piles are reported in [15]. Evidently, the wave propagation velocity depends strongly on the depth of the propagation path between the points of emission (shot) and reception of the elastic wave. Additionally, it is reported in [15] that in preparing a sample from an unconsolidated material, in order to study the velocity of sound for example, its physical properties are disturbed. The same question is raised again in the ninth paragraph in Section 11. 
It was pointed out in Section 4 that the stiffness of a grain column is proportional to the areas of the contact shear bands. In the context of the present computation routine, the frequencies, $f_{i}$, are doubled when the ratio $\bar{d} / R_{b}$ is halved. In this sense, the set of values: $f_{p}=67, f_{1}=791, f_{2}=1603, f_{3}=2561$, etc were realized by assuming that $c_{p}(1)=25$ $\mathrm{m} / \mathrm{s}, c_{p}(25)=15 \mathrm{~m} / \mathrm{s}$, with $\bar{d} / R_{b}$ increasing linearly between the corresponding values of 45 and 150. Thus, the troublesome value of $1 \mathrm{~m} / \mathrm{s}$ for $c_{p}(25)$ can be removed while the column propagation velocity still acquires the realistic value of about $50 \mathrm{~m} / \mathrm{s}$.

\section{The single spring model}

In the study by Nishiyama and Mori [24], the authors attempt to explain the acoustic emissions from an impacted sand bed in terms of a single short spring action below the pestle. Towards this end, rods and disks of various diameters and lengths were pushed or dropped on the sand bed. There is an attempt to show that the dominant frequency, $f_{d}$, decreases with the rod mass, $M$, as the inverse of $\sqrt{M}$. In particular brass rods (disks) with diameter $D=2.5$ $\mathrm{cm}$ and weight as low as $10 \mathrm{gr}$ were used. From a private communication with the principal author, it follows that the shorter rods (disks) were pushed rather than dropped freely on the grain bed. For a rod with $M=100 \mathrm{gr}$, the mass $M_{p}$ on top of a grain column, $\bar{d}=0.35 \mathrm{~mm}$,

amounts to $200 \times 10^{-7} \mathrm{~kg}$, while, in this study, in the case of the $11 \mathrm{~mm}$ steel sphere dropped on the grain bed, $M_{p}=162 \times 10^{-7} \mathrm{~kg}$. The grain column mass is, $M_{c}=16.0 \times 10^{-7} \mathrm{~kg}$ for $N=24$.

The inverse square root relation can be seen in Fig. 4 in [24], up to $M=250$ gr, when the singing sand was placed in a porcelain mortar, $10 \mathrm{~cm}$ in diameter at the top but only about $4 \mathrm{~cm}$ in sand depth. The frequency decreased from about 1000 to $200 \mathrm{~Hz}$ when the rod mass was increased from 10 to $250 \mathrm{gr}$. A similar decrease of $f_{d}$ with rod mass $M$ can be seen in Fig. 6 in [24], for $M<300$ gr. In that experiment, singing sand grains and also silent glass beads, $0.6 \mathrm{~mm}$ in diameter, were placed in the same porcelain mortar, but with only $1.0 \mathrm{~cm}$ in grain depth. It can be seen in [1] that in such confined geometry, all grains become singable to some extent. However, when the sand was impacted in a relatively large container, $40 \times 40$ by $30 \mathrm{~cm}$ deep, $f_{d}$, decreased from about 1000 to $500 \mathrm{~Hz}$, when $M$ was increased from 10 to $100 \mathrm{gr}$, and then it leveled-off for larger $M$, Fig. 2 in [24].

The single spring model can be incorporated into the present approach by identifying the 
dominant frequency, $f_{d}$, with the pestle frequency, $f_{p}$, instead of with the fundamental, $f_{1}$. Thus, with $N=24, d_{j}=\bar{d}=0.35 \mathrm{~mm}, c_{p}(1)=350 \mathrm{~m} / \mathrm{s}$ and $c_{p}(25)=160 \mathrm{~m} / \mathrm{s}$, the eigenfrequencies were, $f_{p}=815 \mathrm{~Hz}, f_{1}=10115 \mathrm{~Hz}, f_{2}=20173 \mathrm{~Hz}$ etc. Roughly, the same frequencies could be

obtained with a somewhat lower value of $c_{p}(1)$ and a somewhat larger value of $c_{p}(25)$. Thus, there is only one frequency envelope centered at $f_{p}$, since the frequencies, $f_{1}, f_{2}$ are far beyond the range where the stick-slip effect would be applicable. The frequency $f_{p}$ decreases exactly as the inverse $\sqrt{M_{p}}$, until it becomes practically zero, while $f_{1}, f_{2}$ hardly decrease. Such a value of $c_{p}(1)$, implying a column propagation velocity, $V_{c} \approx 700 \mathrm{~m} / \mathrm{s}$, seems to be in contradiction with reported experimental values discussed in the previous section. The model would be a possible alternative if there were no harmonics of $f_{d}$, however, this is not the case as will be outlined in the next section.

In the context of the present approach, the decrease of $f_{d}$ with cylinder mass, $M$, when the sand was impacted in a mortar with sand depth about $1 \mathrm{~cm}$, could be justified as follows: For relatively small $M$, there would be the usual number of layers, $N$, and the usual frequency, $f_{d} \approx 1000 \mathrm{~Hz}$. But, for larger $M$, there would be appreciable slippage at the bottom of the mortar and $f_{d}$ would be defined primarily by the phase velocity, $c_{p}(1)$, resulting in significant decrease of $f_{d}$ with mass $M$. It was determined that with $N=12, f_{d}$ decreased from about 1000 to $300 \mathrm{~Hz}$ when $c_{p}(1)$ was decreased from 18 to $18 / 64 \mathrm{~m} / \mathrm{s}$. However, it is reasonable to assume that $c_{p}(2)$ would also decrease with $M$, and that would result in even lower $f_{d}$.

The initial decrease of $f_{d}$ with $M$ when the sand was impacted in the large container, $40 \times 40$ by $30 \mathrm{~cm}$ deep, in [24], could be attributed to an increase of $N$ with increased cylinder mass $M$ before a saturation value of $N$ was reached. When $13 \mathrm{~mm}$ aluminum cylinders, ranging in length from 2 to $12 \mathrm{~cm}$, were dropped from a height of about $10 \mathrm{~cm}$ on the Brevort River sand in the $46 \times 28 \mathrm{~cm}$ by $10 \mathrm{~cm}$ deep container, there were no significant changes in $f_{d}$. However, the $13 \mathrm{~mm}$ aluminum cylinders would have sunk into the sand appreciably more than the $25 \mathrm{~mm}$ brass disks (rods) used in [24].

\section{Experimental results and implications}

The structure of the frequency spectrum of the signal emitted when a bed of singing grains is impacted by a pestle, or a freely falling object, depends on such parameters as: shape, size, surface texture, and speed of the impacting object, wall effects if the impactor is close 
to the walls of the container, and on the history of the grain bed, i.e. exposure to humidity and to previous impacts. The scope of this study is not to determine the effect of any of such parameters on the spectrum structure and in particular on the dominant frequency, $f_{d}$, but to develop a realistic theoretical model that would be based on the grain-grain interaction and would account for the spectral properties of isolated events. It would also account for changes of the spectral properties with one of the parameters listed above, when such changes have been reported in the literature. Thus, in the previous section, the model is used to explain the significant decrease of $f_{d}$ with the mass of the impacting object when it was only a few $\mathrm{mm}$ away from the container wall when the sound was emitted. Furthermore, in the case of moist singing sand emitting sound with, $f_{d} \approx 2500 \mathrm{~Hz}$, Brown et al. [25], it can be argued that the sand mass was relatively very stiff and the grain columns were very short. Similar attempts will be made below regarding the sound from pushed (squeezed) booming dune sand, or the sound accompaning the flow of the same sand through a funnel. In this sense, the use in this study of impacting objects of varying geometry and mass is justified, and in the same sense, the precise value of $f_{d}$ in a given event is not an issue of importance.

In the early stages of this study, Fall 2010, the sand was placed in a ceramic flower pot with $20 \mathrm{~cm}$ rim diameter and $10 \mathrm{~cm}$ depth. A regular pin microphone was placed about $10 \mathrm{~cm}$ roughly above and to the side from the point of impact, while the geophone was placed near the edge of the bed that was about $8 \mathrm{~cm}$ deep. Following an impact, the sand was poured into another container and then back into the flower pot, which was bounced slightly for better grain consolidation. More recently, Fall 2011, the sand was placed in a larger plastic container, $46 \times 28 \mathrm{~cm}$ by $10 \mathrm{~cm}$ in sand depth, with a regular thick towel placed inside the container before the sand was poured so as to prevent any slippage between the sand and the container walls. In such a large container, it was possible to effect several impacts, about $10 \mathrm{~cm}$ apart, before the sand was moved about in the container, with a small plastic cup, and finally the sand surface was leveled and the container bounced slightly. The Geo Space Corporation geophone is omni-directional with natural frequency equal to $14 \mathrm{~Hz}$ suited for detecting relatively low frequency vibrations. The signals were processed by the NI USB-6210 analogue to digital converter and analyzed by the Lab-View Signal Express software of National Instruments. 


\subsection{Impacted singing sand grains}

Fig.2. Frequency spectrum and the microphone recorded signal when an $11 \mathrm{~mm}$ steel sphere was dropped, height $H \approx 10 \mathrm{~cm}$, on a Brevort River singing sand bed in a ceramic flower pot, $20 \mathrm{~cm}$ rim diameter by $10 \mathrm{~cm}$ in depth. $f_{d} \approx 790 \mathrm{~Hz}$.

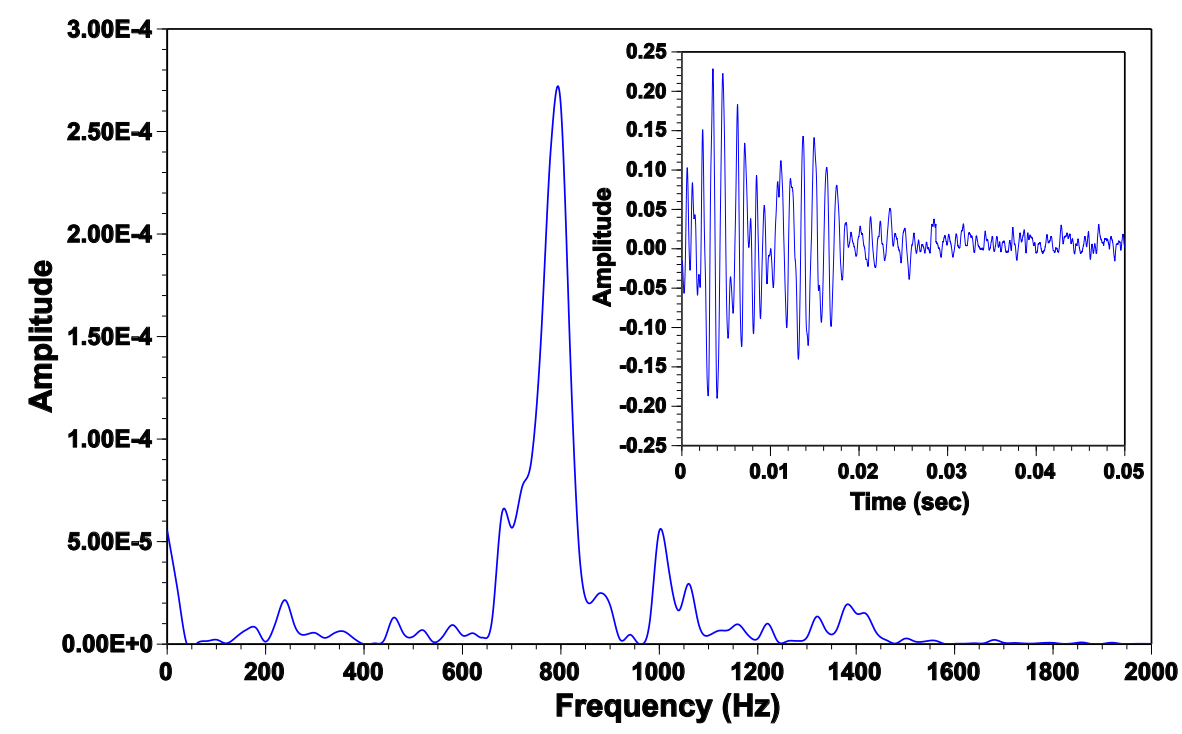

Fig.3. Same as in Fig. 2 but for the geophone signal. The geophone was placed near the pot rim about half immersed in the sand. $f_{d} \approx 770 \mathrm{~Hz}$.

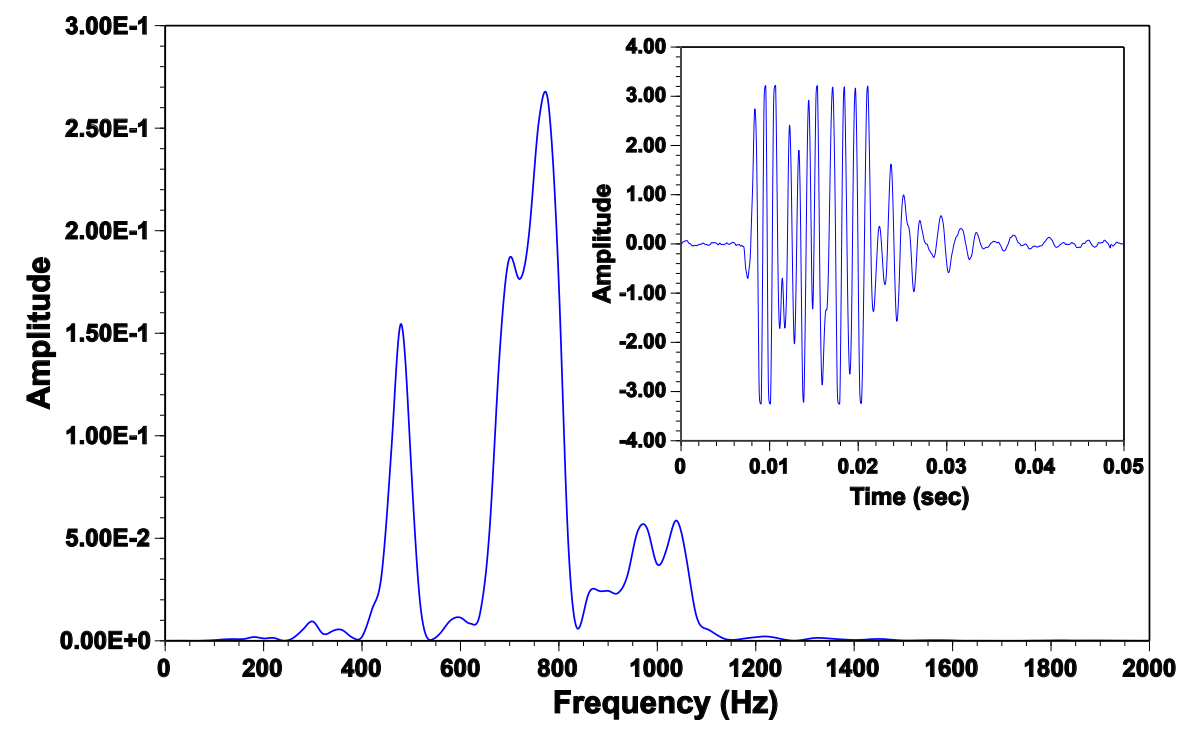

The purpose of this subsection is to demonstrate that harmonics of the fundamental are excited when the grain bed is impacted by small steel spheres and by a glass rod, and thus, 
the single spring model is not applicable in these cases. Furthermore it is shown that the frequency plots are fairly well reproducible even over long time periods.

The same recordings were repeated recently, about one year later, with the same sand in the large plastic container. The microphone plots were reproduced quite well overall, however, that was not the case for the geophone plots, where, the slight elbow at about $695 \mathrm{~Hz}$ would appear as a totally separate peak. It could be argued that the geophone signal from the relatively small sphere is more susceptible to localized grain mass un-isotropies as the sphere was well immersed in the sand after impact. The peak at about $475 \mathrm{~Hz}$ would be an indication of such an effect. It is likely due to the increase of the period of the inset signal after about 22 ms, implying that near the end of the impact, there was an appreciable increase in the grain column length in the slip shear band.

From the simple Fourier transform theory, the half width of the transform of the inset signal with length, $\Delta t=18 \mathrm{~ms}$ in Fig. 2 , is $56 \mathrm{~Hz}$, and the separation of the side peaks from the center of the main envelope is $1.5 \times 56=84 \mathrm{~Hz}$. The half width of the main envelope is about $62 \mathrm{~Hz}$, and the separation of the first side peak (elbow) on the right from the center, at $877 \mathrm{~Hz}$, is $87 \mathrm{~Hz}$. However, the separation of the first elbow on the left at $628 \mathrm{~Hz}$ amounts to $162 \mathrm{~Hz}$, implying that it is not a Fourier side peak, and since it is also present in Fig.3, it could not be argued that it is due to some sort of noise, but rather due to a separate slip shear band.

The question of noise is raised below in connection with Fig. 15. Here, it could be defined as follows: It arises from the incoherent superposition of the elastic wave-trains emitted as a grain rubs its way past other grains, and of the wave-trains due to vibrations in grain columns of random length and direction around the pestle. Additionally, there would be low frequency content due to air mass accelerations recorded by the microphone. In this sense, the envelope at around $1000 \mathrm{~Hz}$, in both plots, could be attributed to random column vibrations around the sphere, or due to such vibrations in a relatively thin and ill-defined slip side band. In this sense, when the slip shear band becomes totally ill-defined, then it becomes a source of noise. Thus, there could be two or more slip shear bands around the lower hemisphere of the impacting sphere, and around the pestle in general. A slight rotation of the sphere during the impact, or a non-vertical direction of the pestle, would be a cause of such multiplicity of slip shear bands. The lack of harmonics in the above plots could be attributed to the 
relatively short duration of the event and the lack of sufficient energy for the excitation of the corresponding modes.

Fig.4. Frequency spectrum of the microphone recorded signal when a $16 \mathrm{~mm}$ steel sphere was dropped on the Brevort River singing sand in the large plastic container, $46 \times 28 \mathrm{~cm}$ by $10 \mathrm{~cm}$ sand depth, from the height $H \approx 20 \mathrm{~cm}$. The vertical range was reduced somewhat in order for the first harmonic of $f_{d} \approx 578 \mathrm{~Hz}$ to be seen at about $1175 \mathrm{~Hz}$. The second could be seen with further reduction at about $1780 \mathrm{~Hz}$.

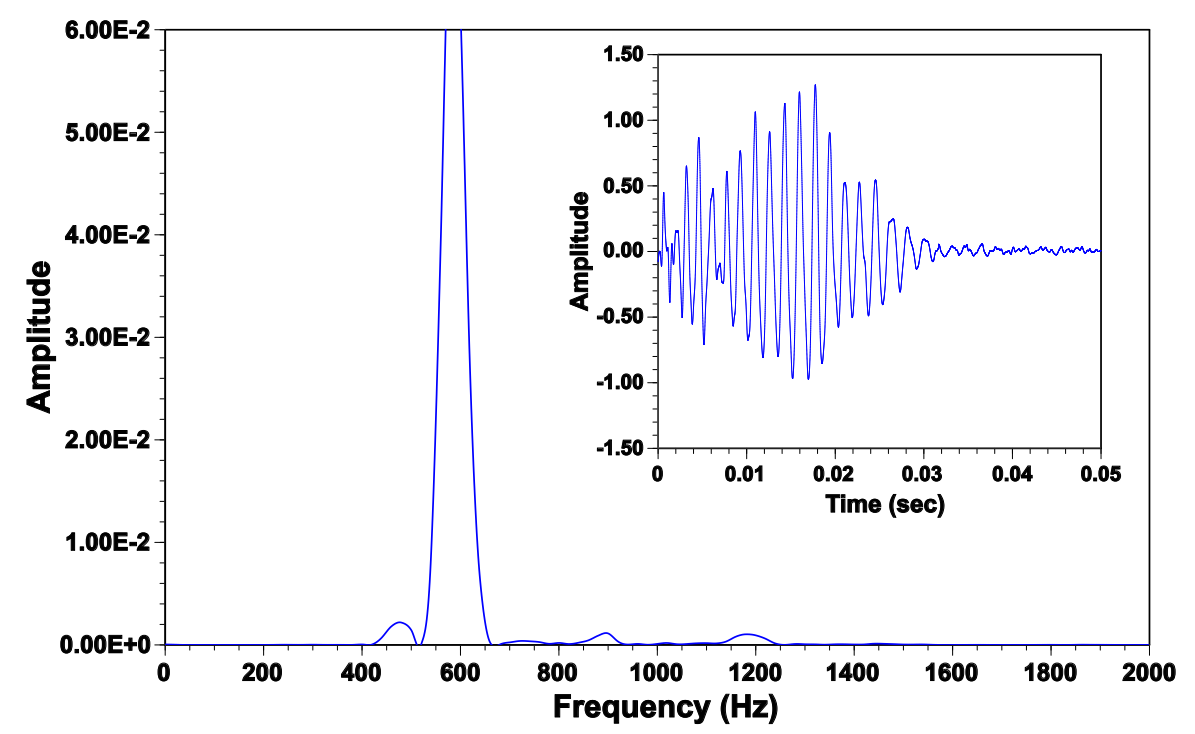

Fig.5. The same as in Fig. 4 but for the geophone recorded signal. $f_{d}$ is estimated at about $590 \mathrm{~Hz}$. The second harmonic at just under $1800 \mathrm{~Hz}$ is discernible.

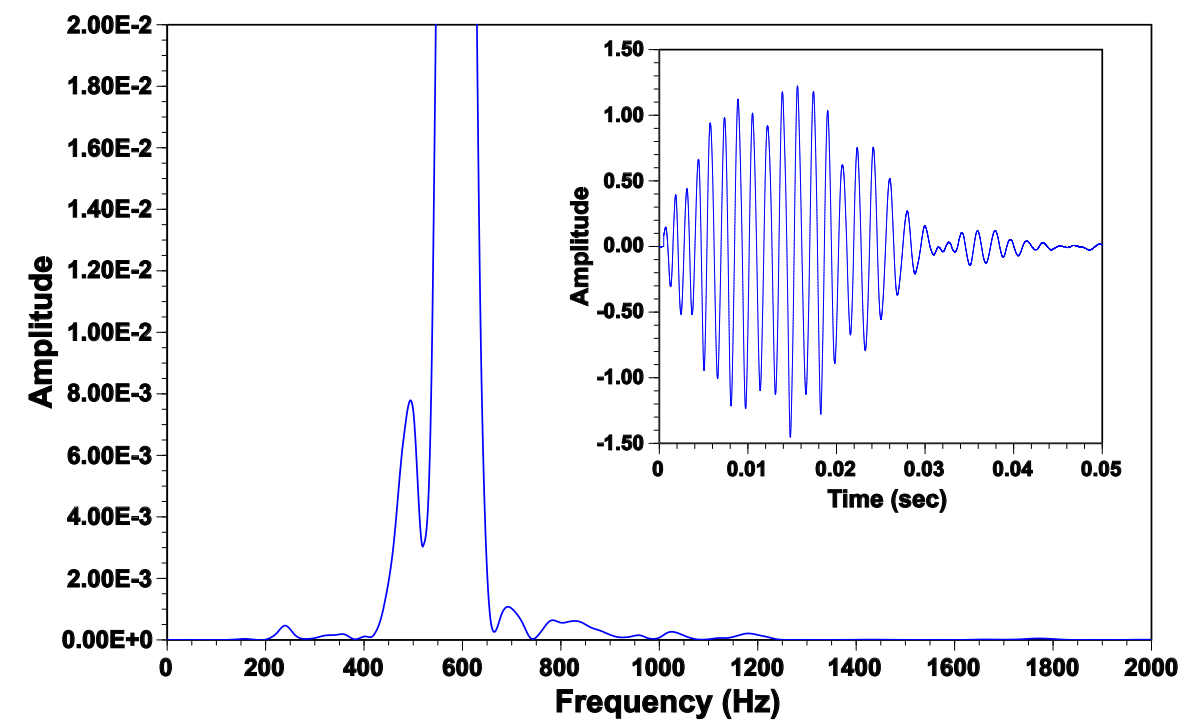


Fig.6. Same as in Fig. 4 but for a $25 \mathrm{~mm}$ steel sphere. $f_{d} \approx 467 \mathrm{~Hz}$ and the second harmonic is barely seen at about $1400 \mathrm{~Hz}$.

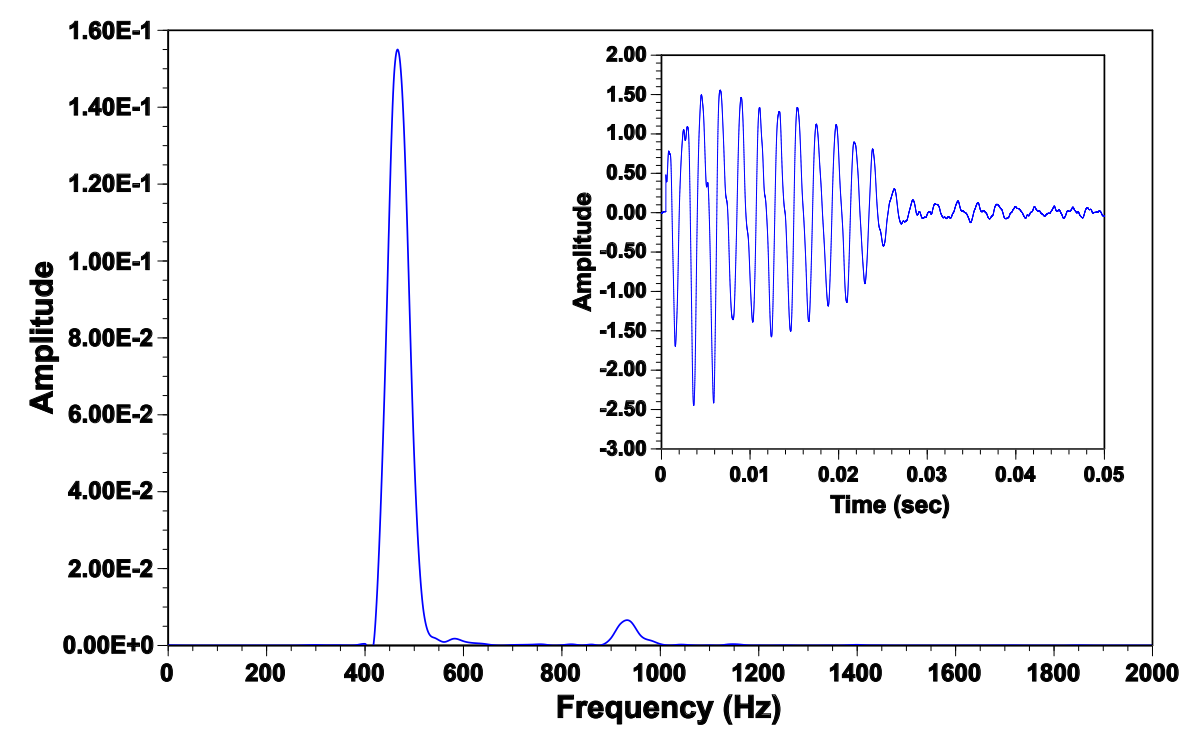

Fig.7. same as in Fig. 6 but for the geophone signal. The first harmonic of $f_{d} \approx 467 \mathrm{~Hz}$ is quite prominent and the second is visible. The decrease of $f_{d}$ from $590 \mathrm{~Hz}$ in Fig. 5 to 467 $\mathrm{Hz}$ in Fig. 7 could be attributed to the increase in the column number $N$.

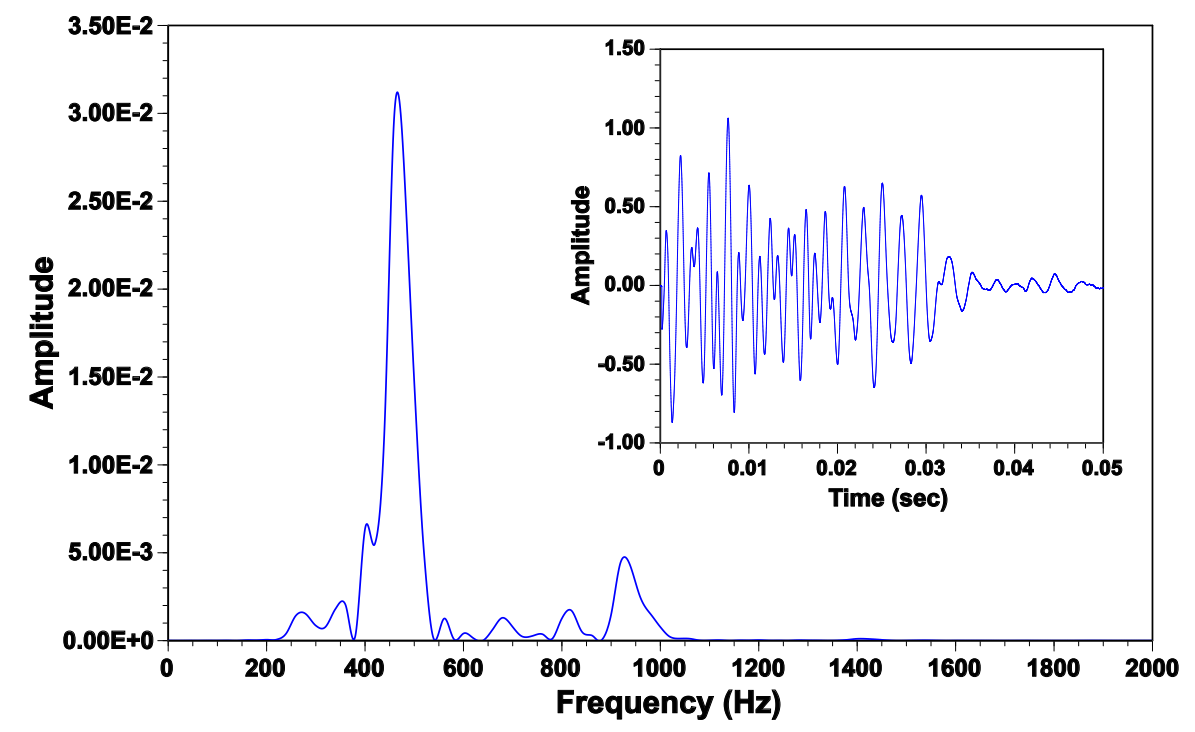


Fig.8. Frequency spectrum of the microphone recorded signal when a glass rod, length 7.5 $\mathrm{cm}$, diameter $1.5 \mathrm{~cm}$ obtained from the Museum of Sand in Nima, Japan, was pushed manually into the Brevort River singing sand bed in the large plastic container, $f_{d} \approx 630 \mathrm{~Hz}$. The side peak at $700 \mathrm{~Hz}$ is a Fourier side peak. The harmonics at about 1250 and $1870 \mathrm{~Hz}$ are discernible.

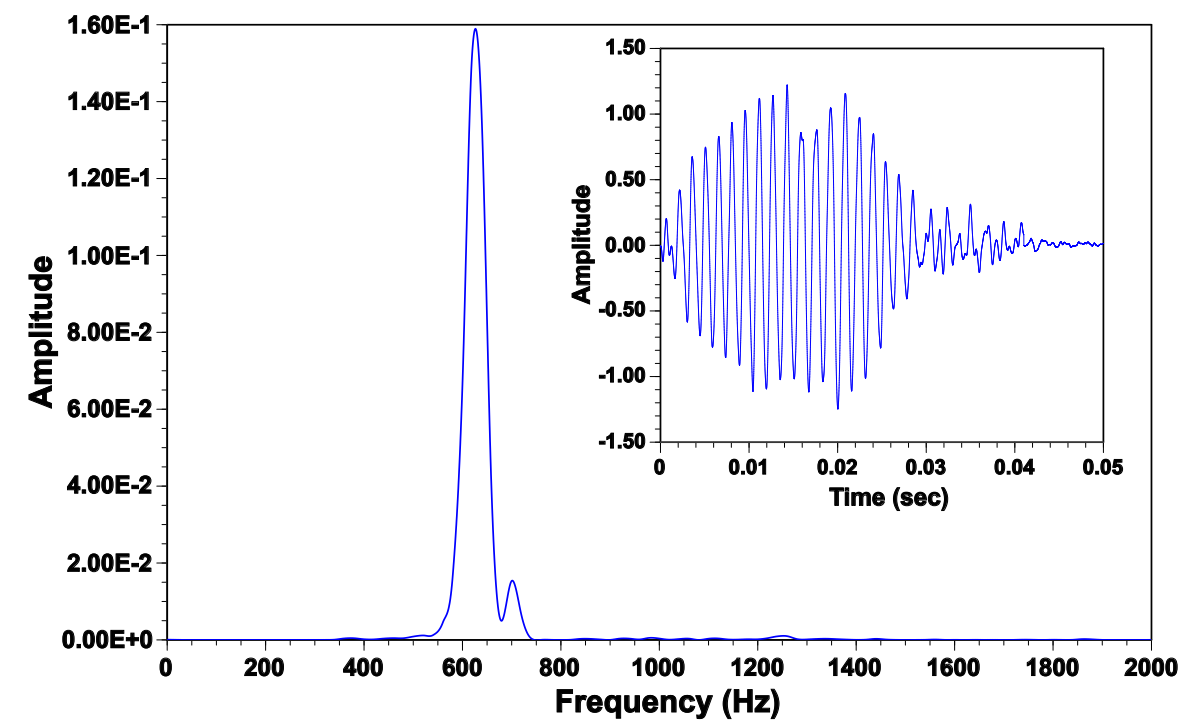

Fig.9. same as in Fig. 8 but for the geophone signal. It is remarkable that the two spectra are practically identical in this case.

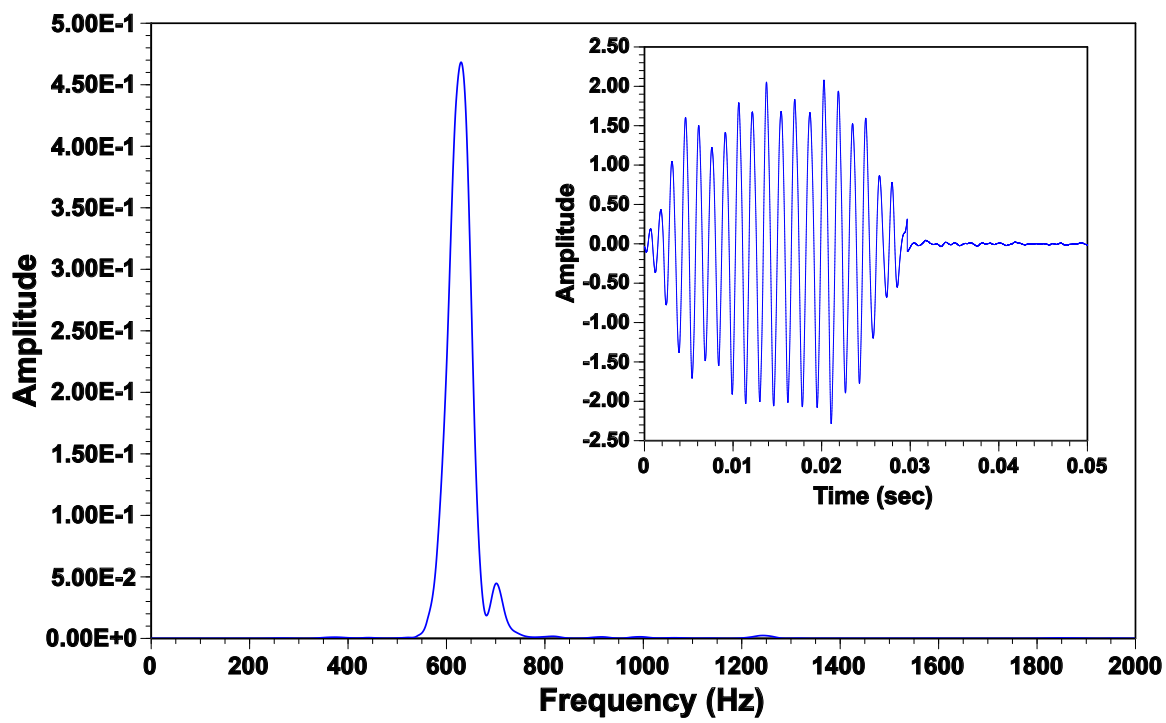


Fig.10. Same as in Fig. 8 but with the sand in a flower pot, with $20 \mathrm{~cm}$ rim diameter and $10 \mathrm{~cm}$ in depth, nearly one year before the recording of the signal in Fig. 8. One harmonic is clearly seen at $2 f_{d}$ with $f_{d} \approx 590 \mathrm{~Hz}$.

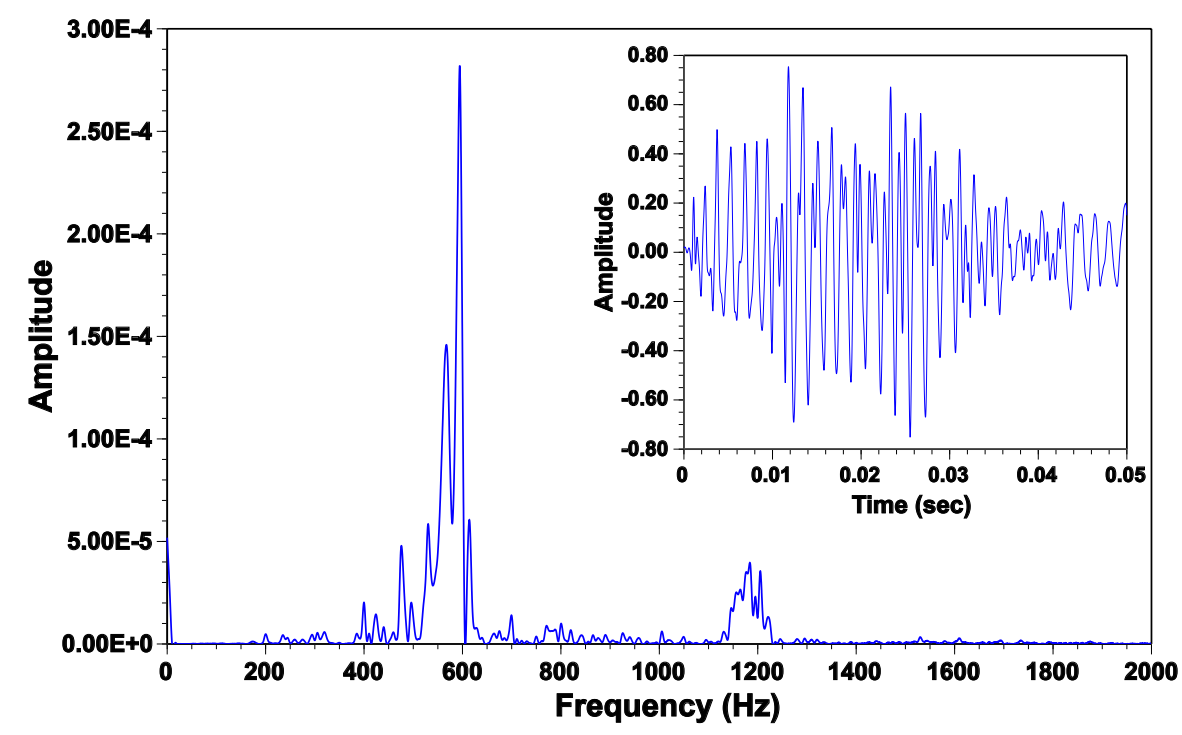

Fig.11. Same as in Fig. 10 but for the geophone signal. The second harmonic is quite discernible at about $1770 \mathrm{~Hz}$ with $f_{d} \approx 590 \mathrm{~Hz}$.

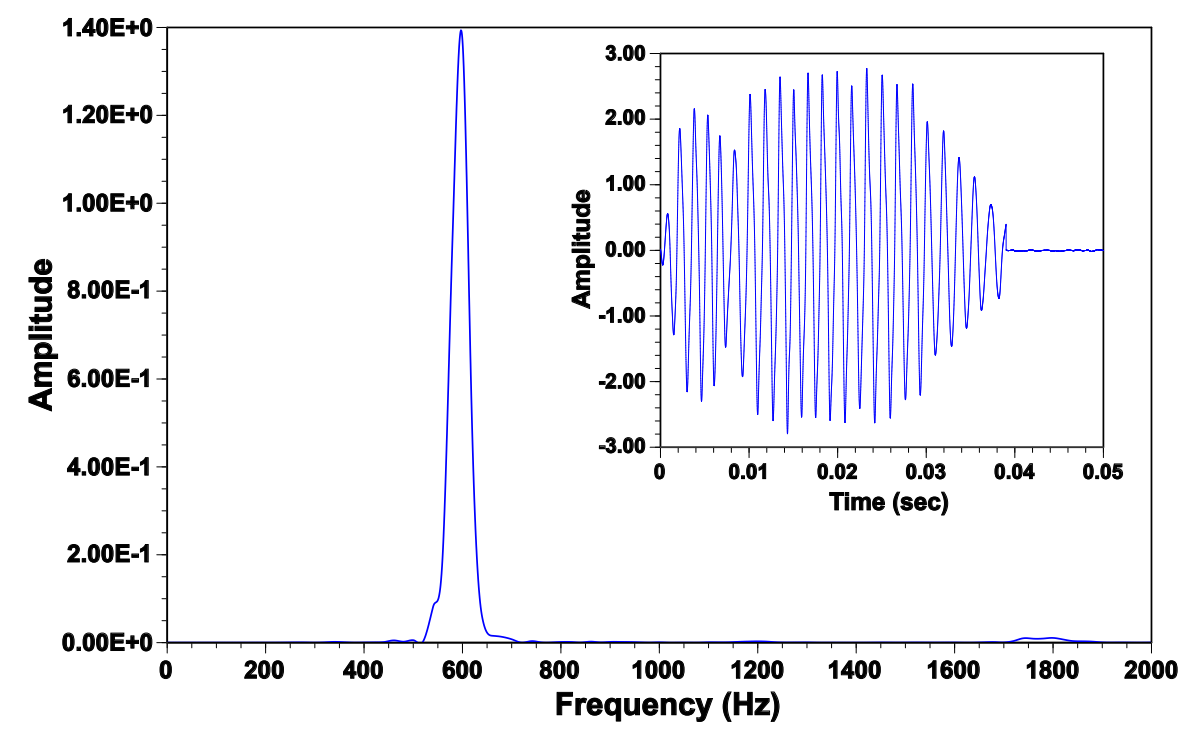

The plots in Figs. 4 to 7 were fairly well reproducible. In these relatively simple geometries, it is safe to argue that the slip shear band is a few mm thick and has the shape of a disk curved around the impacting sphere, with radius roughly that of the sphere [1]. It is also safe to argue that a larger disk radius would imply a larger disk thickness which corresponds proportionately 
to a lower frequency $f_{d}$. From Figs. 4 and 6 , the frequency ratio is $578 / 467=1.24$, while the square root of the radii ratio is, 1.25 . For the case of the 16 and $25 \mathrm{~mm}$ glass spheres, the analogous frequency ratio is, $800 / 600=1.33$. Thus, for these particular cases the slip band thickness increases roughly as the square root of the sphere radius.

The increase of $f_{d}$ from 590 in Fig. 10 to $630 \mathrm{~Hz}$ in Fig. 8, in the course of one year could be due to the aging of the sand. It could also be due to a more or less deviation of the rod axis from the vertical direction. The sand was kept well sealed in plastic bags when not in use. When the same rod was dropped freely and vertically on the same sand bed from the height of about $10 \mathrm{~cm}, f_{d}$ was about $550 \mathrm{~Hz}$, a value definitely lower than $630 \mathrm{~Hz}$ when the rod was hand held, Fig. 8. These results lead to the conclusion that the single spring model cannot be applicable in this case, for when hand held, the effective pestle mass is considerably larger than that of the rod and $f_{d}$ ought to be considerably lower. There is evidence here of the effect of the impact shock on the sand mass when the rod is dropped. It could be argued that it results in longer grain columns and lower $f_{d}$.

The absence of the low frequency content, i.e., at around $f_{p}$, in all the above plots except for the $11 \mathrm{~mm}$ steel sphere, could be attributed to the large energy required for the excitation of the pestle vibration, especially when the pestle is hand held. Thus, when a $16 \mathrm{~mm}$ glass sphere was dropped on the same sand bed, in the large plastic container, from the height of about $15 \mathrm{~cm}$, the geophone frequency spectrum included a weak low frequency content at about $70 \mathrm{~Hz}$. In some signals, more than others, the period increases somewhat with time, suggesting that there is a slight increase of the column number $N$ during penetration. The lack of musicality of the low frequency sound emitted, $f_{d} \approx 250 \mathrm{~Hz}$, when the flat end of a wood rod, diameter about $8 \mathrm{~cm}$, impacted the Brevort River sand could be attributed to the relatively low flowability of the sand. The duration of the signal amounted to only about 25 ms resulting in only about eight oscillations.

There are only two known reports on harmonics of $f_{d}$. In Fig. (c) in [11], $f_{d}=860 \mathrm{~Hz}$ and two harmonics are depicted. However, the impaction process of the "squeaking" sand is not specified. In the report by Takahara [26], the singing sand was impacted by a smooth rounded wood rod in a glass funnel. The size of the rod and the funnel are not specified. The fundamental is, $f_{d}=599 \mathrm{~Hz}$ and four harmonics are depicted. A strong first harmonic at 1045 $\mathrm{Hz}$ was evoked when the Brevort River sand was impacted by the $7.5 \mathrm{~cm}$ glass rod in a regular 
glass cup with diameters of $8 \mathrm{~cm}$ at the rim, $5 \mathrm{~cm}$ at the base, $9 \mathrm{~cm}$ in height and filled to the height of $7 \mathrm{~cm}$. There were also weaker second and third harmonics.

\subsection{Pushed musical grains}

Fig. 12. The frequency spectrum of the microphone recorded signal when the $7.5 \mathrm{~cm}$ glass rod was drawn manually in a nearly vertical position immersed at about $3 \mathrm{~cm}$ along the surface of the Brevort River sand in the large plastic container. The signal is shown only up to $160 \mathrm{~ms}$, but the recorded signal lasted up to $500 \mathrm{~ms}$.

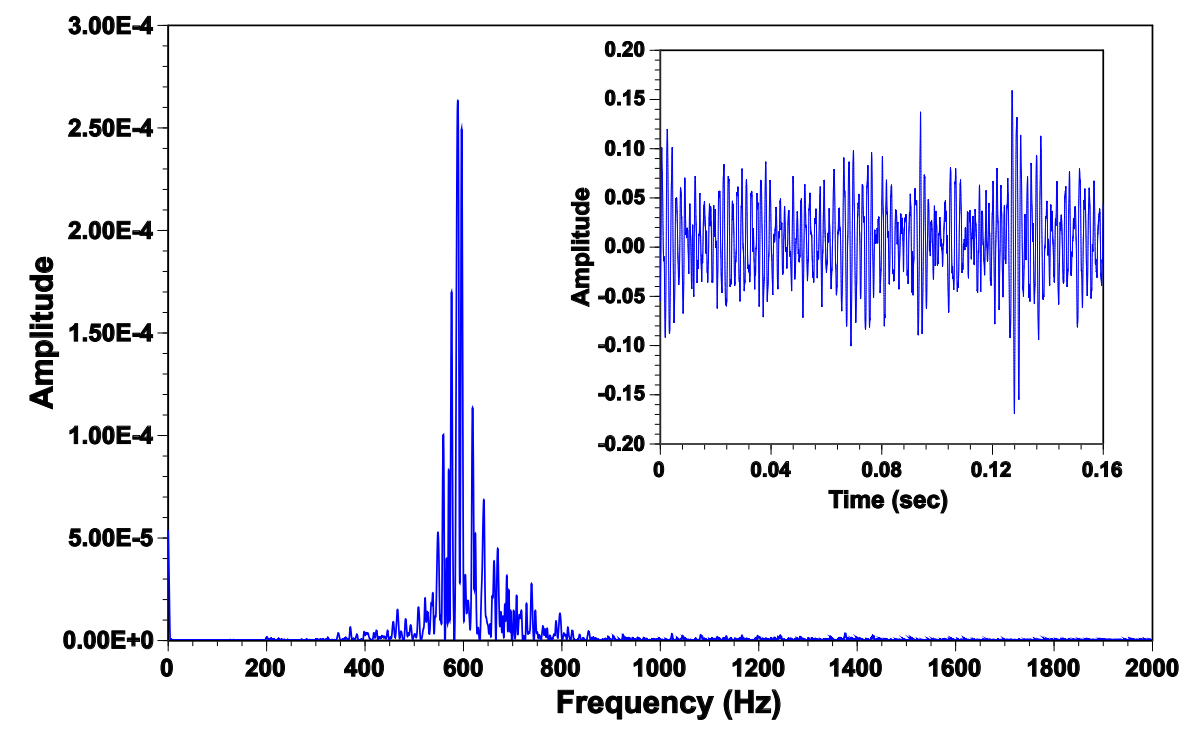

Fig.13. The same as in Fig. 12 but for the geophone recorded signal.

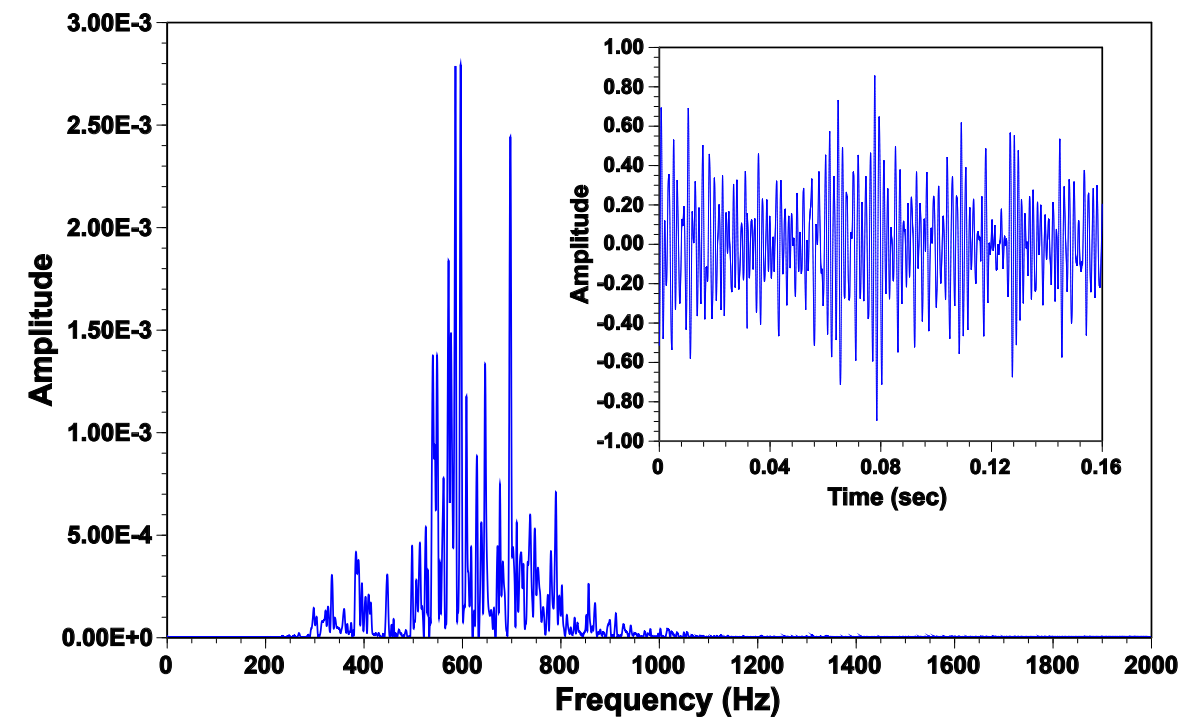


Fig.14. The frequency spectrum of the microphone recorded signal when a $13 \mathrm{~mm}$ wood rod was turned manually inside a $9 \mathrm{~cm}$ glass jar containing silica gel grains with depth of about $10 \mathrm{~cm} . f_{d} \approx 450 \mathrm{~Hz}$.

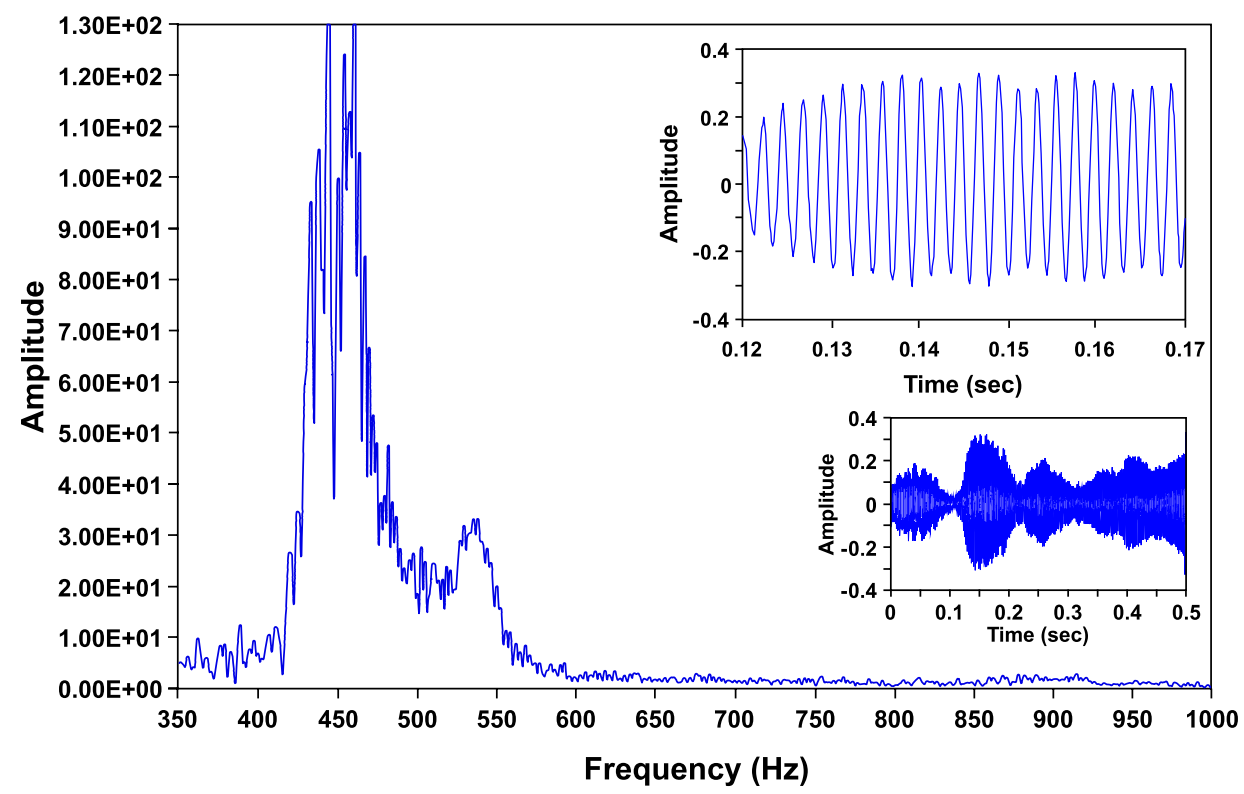

The second minor envelope in Fig. 14 at about $540 \mathrm{~Hz}$, could be due to a minor slip band possibly near the bottom of the rod. It could correspond to the sharp peak at about $675 \mathrm{~Hz}$ in Fig. 13. The signal insert from 0.12 to $0.17 \mathrm{~s}$ happens to be quite monochromatic.

The plots in Figs. 12 and 13 demonstrate that a continuous musical sound, similar to that from an avalanching sand dune, can be emitted by the singing (squeaking) sand grains when sheared (squeezed) in a continuous manner. The spectrum envelope in Fig. 12 resembles quite well that in Fig. (a) in [11], and that seen in a YouTube presentation by the authors of [4], with the title, Booming Sands, and narrated by M. Hunt. The ruggedness of the frequency spectrum, as opposed to that in Fig. 6 for example, can be attributed to the continuous renewal of the slip shear band in front of the rod in the course of time. Similar plots were prepared, but not shown, when the same sand was similarly sheared by a $13 \mathrm{~mm}$ wood rod. The signals were considerably more noisy and the frequency envelopes considerably more rugged and wider than in Fig. 12. Evidently, the smooth surface texture of the glass rod, as opposed to the wood rod, resulted in considerably more smooth transition from one slip shear band to the next. 


\subsection{Impacted silent sand grains}

Fig.15. Frequency spectrum and the microphone recorded signal when a $13 \mathrm{~mm}$ wood rod was tapped (pushed) into a bed of local silent beach sand in the large flower pot, $20 \mathrm{~cm}$ at the rim and 10 deep. The weak hissing-like sound was barely audible

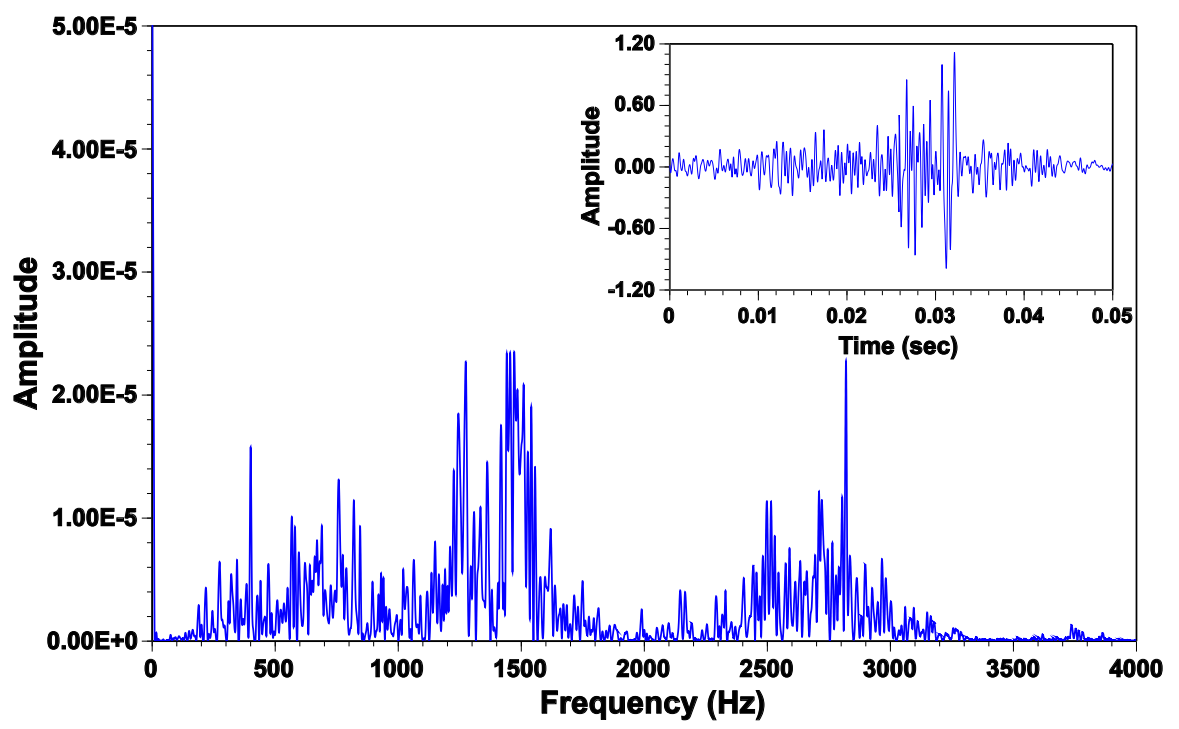

Fig.16. Same as in Fig. 15 but for the geophone recorded signal. $f_{d} \approx 457 \mathrm{~Hz}$.

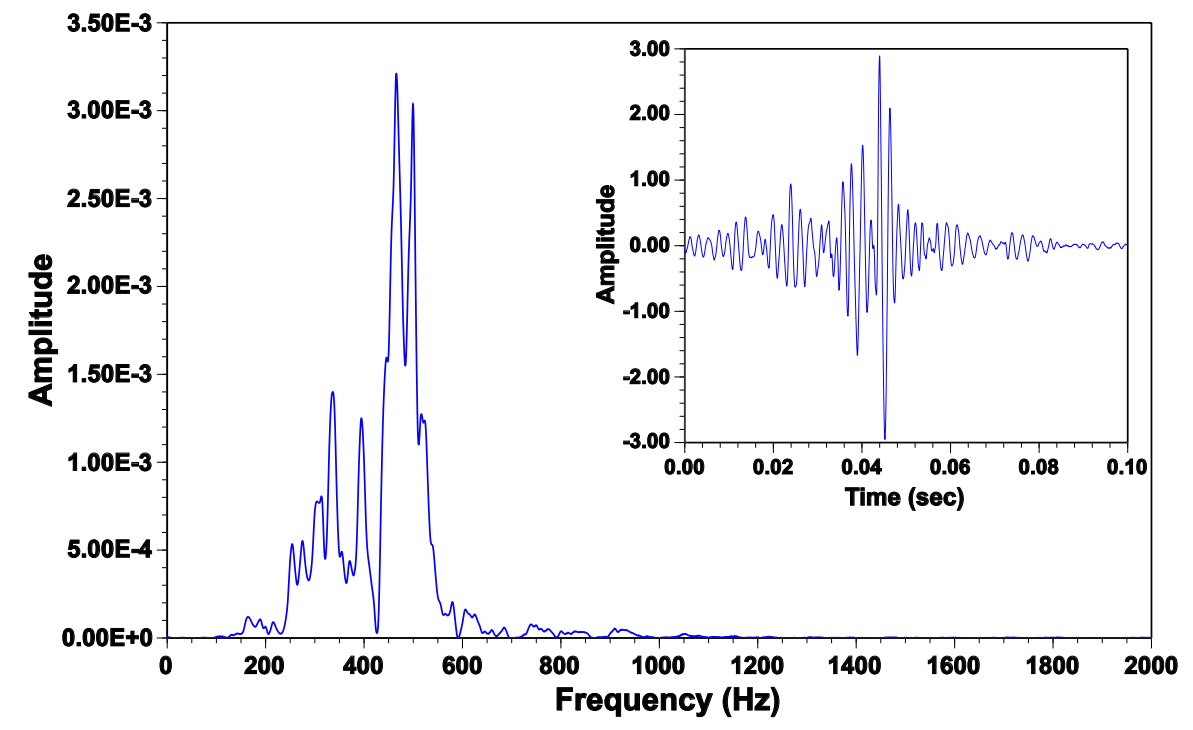

The frequency spectrum shown in Fig. 15 is typical of silent sand beds impacted by a variety of pestles. The noise envelope between about 2400 and $3200 \mathrm{~Hz}$ is nearly omni-present for 
all sorts of pestle and grains, suggesting that it originates with grain-grain rubbing near the pestle where the stress forces are maximum. Effectively, these frequencies could correspond to grain asperity collisions as the grains slide past one another. The rest of the noise frequency content could be attributed to grain column vibrations near and parallel to the surface. The grain number, $N$, varies randomly and the vibrations are nearly incoherent. This is not the case for the grain columns below the pestle, resulting in a nearly monochromatic vibration in some cases.

There is considerable variability of the spectrum shown in Fig. 16. In some cases, the envelope is wider comprising many peaks, suggesting that the slip shear band is less well defined, comprising a wider range of grain column numbers $N$, and in some cases it is narrower without the side peaks between 200 and $400 \mathrm{~Hz}$, for example. However, the noise level approximately above $700 \mathrm{~Hz}$ is nearly absent in all cases. A wave with frequency $f=1000$ $\mathrm{Hz}$, propagating along the surface with velocity $V_{s}=1 \mathrm{~m} / \mathrm{s}$, has wavelength, $\lambda=1.0 \mathrm{~mm}$, not appreciably larger than the average grain diameter, $\bar{d}=0.35 \mathrm{~mm}$. Thus, the wave propagation is likely to be cut-off or the wave amplitude to be reduced substantially.

Figures 17 and 18 correspond to Figs. 15 and 16 except that the rod was tapped (pushed) into a bed of crusher dust, used as road surface cover in place of pavement. Most of the fine dust had been removed. The use of the relatively small diameter wood rod was necessitated by the large particles in the bed, i.e., a larger diameter rod would not penetrate into the bed smoothly. Despite the unusual crusher dust grain size distribution, the sound was appreciably louder and somewhat more musical than from the silent beach sand. When the same crusher dust bed was impacted by one arm of a walnut cracker, a metallic rod $8 \mathrm{~mm}$ in diameter tapered to a smooth rounded end, the microphone noise frequency content around 3000 and $1500 \mathrm{~Hz}$ was reduced relatively to that around $500 \mathrm{~Hz}$, while the geophone spectrum was similar to that in Fig. 18. 
Fig.17. Frequency spectrum of the microphone recorded signal when a $13 \mathrm{~mm}$ wood rod was tapped (pushed) into a bed of crusher dust in the large flower pot. The grains were very irregular in shape and varied in size from about $1 \mathrm{~mm}$ in overall diameter to as large and irregular as $10 \times 5 \times 2$.

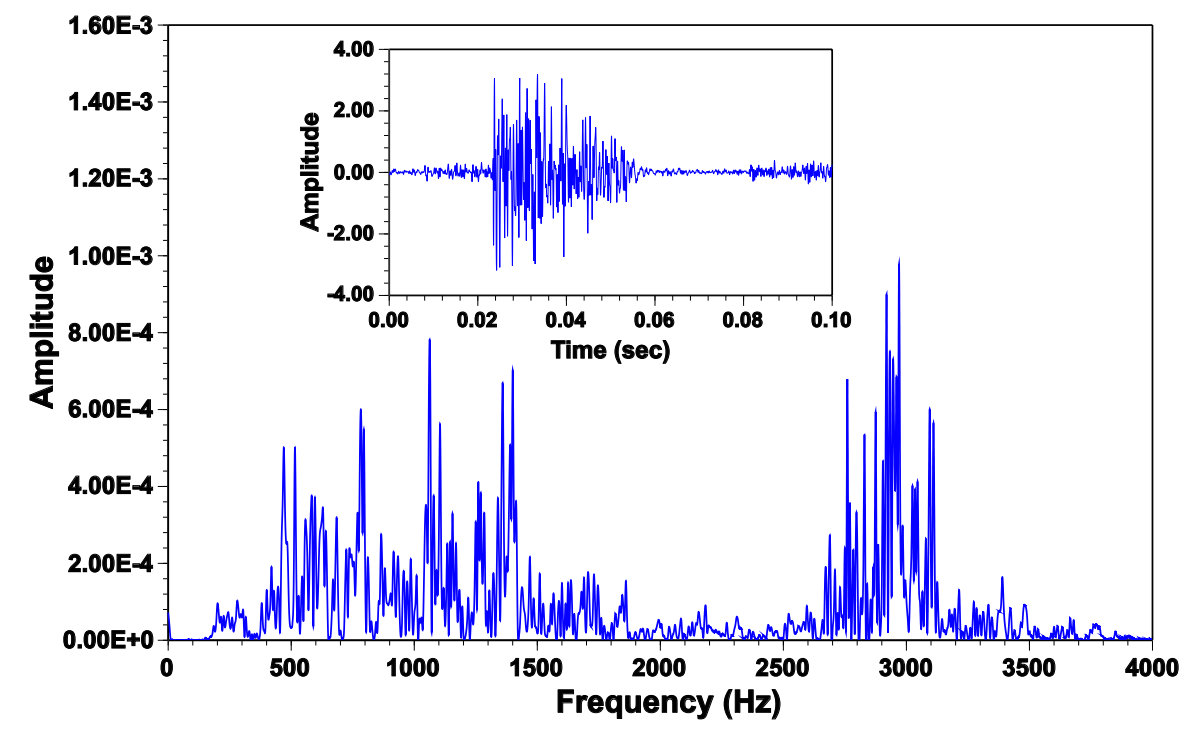

Fig.18. Same as in Fig.17 but for the geophone recorded signal. $f_{d} \approx 362 \mathrm{~Hz}$.

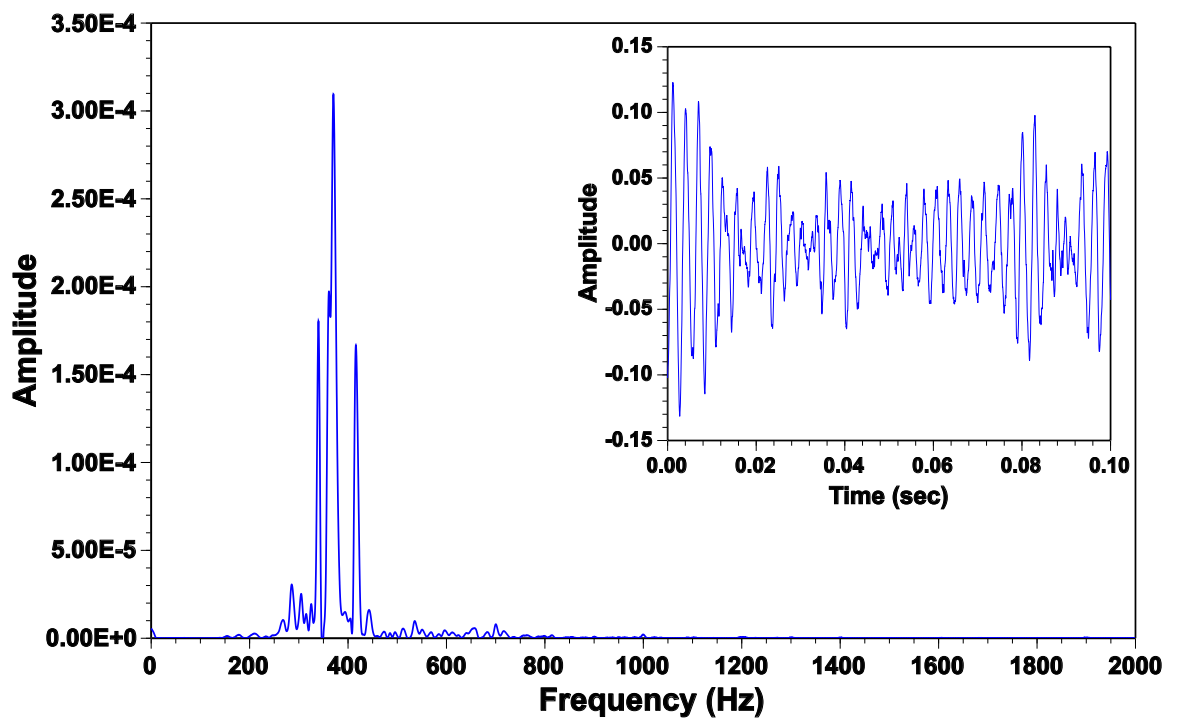


Fig.19. Frequency spectrum of the microphone recorded signal when a metallic rod, $4.2 \mathrm{~mm}$ in diameter, was pushed into the same bed of crusher dust in the large flower pot. $f_{d} \approx 435$ $\mathrm{Hz}$.

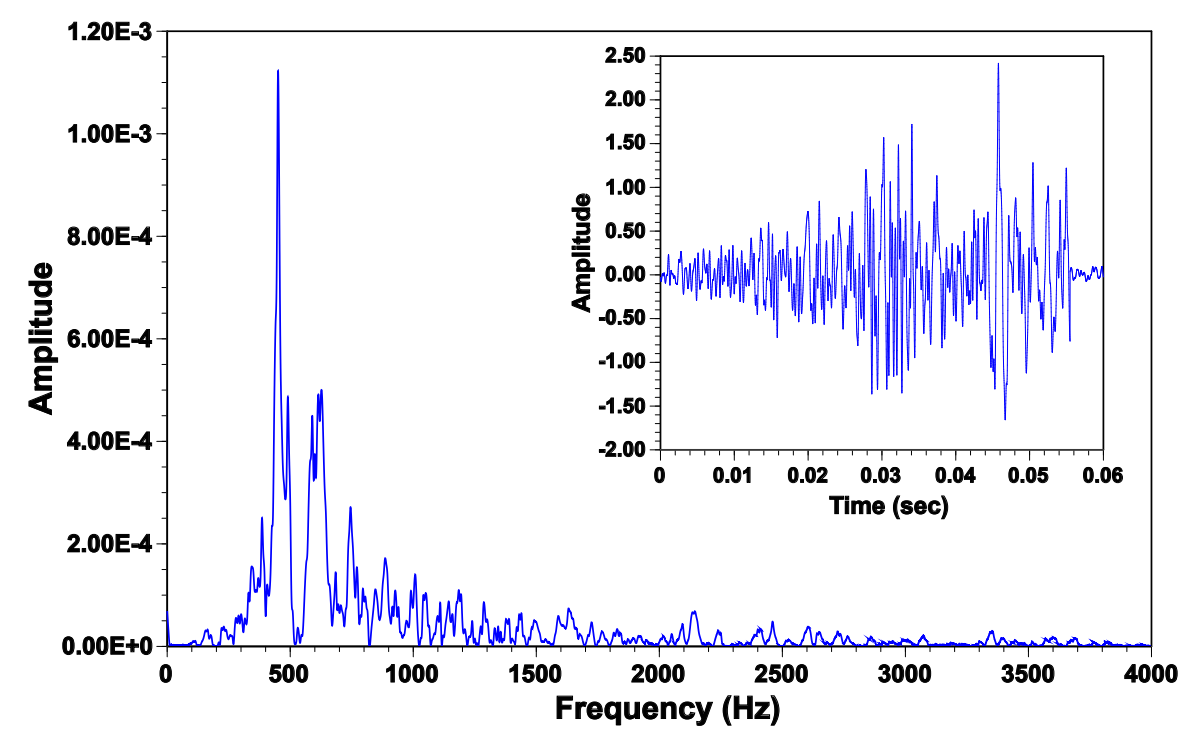

Furthermore, when the same grain bed was impacted by the nearly blunt end of a walnut scraper, a metallic rod $4.2 \mathrm{~mm}$ in diameter with the end polished smoothly for $10 \mathrm{~mm}$ and then slightly tapered to a conical end, the emission resulted in a geophone spectrum similar to that in Fig. 18 and in a microphone spectrum shown in Fig. 19. Such a spectrum resembles more closely those of the singing as opposed to those of the silent grains. Evidently, the small diameter and the polished end of the pestle resulted in sufficiently low stress level around the leading front of the pestle so as to reduce the excitation of the grain-grain rubbing and the near surface grain column vibration noise to very low levels.

\section{Collective column vibrations and the stick-slip effect}

It now appears safe to conclude that the transition from non-singability to singability of a grain bed is based on two premises: (a) the reduction of the stress level on the grains around the pestle and (b) the transfer of the pestle energy to the grain column vibrations below the pestle. The first could be met if there were sufficient grain-grain slippage in the slip shear band below the pestle that would result in low stress level around the pestle, and the second, if the stick-slip effect would be applicable, i.e., if the friction coefficient would decrease with 
relative velocity between the grains [20].

The degree of grain-grain slippage was tested by dropping a $16 \mathrm{~mm}$ steel sphere from a height of $20 \mathrm{~cm}$ on grain beds in the $20 \mathrm{~cm}$ flower pot composed of: (a) Brevort River sand, (b) Providence Bay, Manitoulin Island, ON, CA, nearly silent sand and (c) a local beach sand sounding even more silent. In case (a), the sphere was barely visible at the center of the crater about $10 \mathrm{~mm}$ deep, in case (b), nearly $1 / 3$ of the upper hemisphere was visible and in case (c), nearly all of the upper hemisphere was visible. It is estimated that in case (a), the sphere traveled about twice as far as in case (c) after the initial impact.

Then, there is the question of the synchronization of the column vibrations in the slip shear band. Is it due to a wave propagating in the same band as in the case of the freely avalanching dune sand? In the case of the walnut scraper rod, the diameter of the slip shear band would be equal to about $5 \mathrm{~mm}$, while the synchronization wave would have wavelength equal to 10 $\mathrm{cm}$ for propagation velocity and frequency equal to $50 \mathrm{~m} / \mathrm{s}$ and $500 \mathrm{~Hz}$ respectively. Thus, the source of the grain column synchronization has to be sought elsewhere. It could be argued that a few dominant column vibrations would induce the same vibration in the pestle, which in turn would force all columns to vibrate in phase with it, thus, resulting in a collective vibration of all grain columns. In turn, such an intense vibration would facilitate the slipping of the grain mass away from the advancing pestle and would nearly eliminate the surface noise described above. In this sense, the slip band acts like a short dipole radiating waves, with frequency $f_{d}$, in the surrounding grain mass.It is argued in Sections 5 and 8 that inside the contact bands, $\xi_{x} \gg \xi_{z}$ and that, by extension the grain oscillation amplitude along $\hat{x}$ is greater than that along $\hat{z}$. Furthermore, $\xi_{z}$ was required to vanish in (1), at the bottom of the slip shear band. Accordingly, a shear wave would be radiated into the grain mass surrounding the slip shear band.

It could be argued that a minimum (threshold) impact pestle velocity is required for the initial excitation of the few column vibrations. Such thresholds are always present before a musical event can occur. When the wood rod was held vertically and forced to move horizontally through a bed of silica gel grains, the immersion depth had to be more than about $2 \mathrm{~cm}$ and the velocity had to be more than about $20 \mathrm{~cm} / \mathrm{s}$. Moreover, when a plastic bead, $1 \mathrm{~cm}$ in diameter, was buried in a flat pile of the Brevort River singing sand and pulled horizontally by a string, the depth had to exceed about $3 \mathrm{~cm}$ and the string had to be pulled 
rather sharply. Similar thresholds are seen in [9] where the booming dune grains were pushed by a blade.

\section{Grain flowability and grain confinement}

It seems fair to argue that what distinguishes primarily a freely avalanching booming sand, from other silent avalanching sands, is the relatively high avalanche front and the apparent

high flowability of the grains. Reference to high flowability, where the sand flow is compared to that of a water stream, can be found in the reports by: Sholtz et al. [27], Bagnold [28] and Humphries [29]. When Brevort River singing sand was placed in a plastic container, $40 \times 35$ by $25 \mathrm{~cm}$ deep and dumped sharply on the side of a nearby dune ridge with slope over $30^{\circ}$, there was no appreciable avalanche front. The sand flow was sluggish and characterized more by plate-like motion than free surface grain motion. It was more sluggish than that of ordinary silent sand motion when similarly dumped. However, when a cupful of sand was tossed with some force at an angle of about $45^{\circ}$ on the flat top of a sand pile, the usual sound was evoked.

In [16], it is reported that when booming grains were placed in a sealed glass jar, $17.5 \mathrm{~cm}$ in length by $10 \mathrm{~cm}$ in diameter, half full, and rapidly tilted, "a violent roar" could be produced. Similarly, when Brevort River singing sand was placed in a glass jar, $17 \mathrm{~cm}$ in length by $8 \mathrm{~cm}$ in diameter but only $7 \mathrm{~cm}$ at the lip, and then tilted sharply, no sound was produced until most of the sand had flown out of the jar and the sand height above the lip was about 15 mm. During the sound emission, the sand appeared to flow out of the jar as in one piece, thus, reinforcing the concept of the slip shear band adjacent to the jar lip.

Similarly, when booming sand from Sand Mountain in NEV. USA, available in the laboratory of the authors of [10] some 20 years ago, was placed in a large plastic funnel, $15 \mathrm{~cm}$ rim diameter and $17 \mathrm{~mm}$ exit end, a low frequency vibration sound estimated at under $100 \mathrm{~Hz}$ could be heard. However, when the same was attempted with the Brevort River singing sand, no sound was evoked. Thus, it could be argued that singing sand grains do not boom because of their low flowability.

It is not clear what caused the "violent roar" sound when the glass jar was rapidly tilted. It could be due to column vibrations in a slip shear band on the surface, as outlined in the next Section, or due to a similar band adjacent to a glass wall. However, on page 45 in [16], it is reported that the hum sound was emitted when the booming sand was poured from a 
hopper into a bag. Thus, a free avalanche booming was produced in the laboratory unless the hum sound was due to the impact of the flowing sand on the stationary sand in the bag. However, a cursory analysis of the sound emitted when the hand held booming sand was dropped on the dune surface, revealed a sound with $f_{d}$ in the neighborhood of $250 \mathrm{~Hz}$. The sound is available in the YouTube video presentation by the authors of [9].

The funnel-like experiment was effected also by Lewis [16] by effectively cutting a square hole into the glass jar cap, about $2.5 \mathrm{~cm}$ in side, and inverting the jar. The ensuing sound is described as "hum". In the context of this approach, the grain columns, somewhat above the plane of the hole, would span the hole width, but the grain contact phase velocity, $c_{p}(j)$, would have minimum value in the middle of the column instead of at the top, as in the case of the impacted bed or in the case of the freely avalanching dune sand.

Booming dune grains do not sing in the sense of emitting a musical sound when placed in a large dish and impacted by a rod with diameter about $2 \mathrm{~cm}$. In the context of this approach, this is so since the relatively high flowability of such grains results in unstable long grain columns. In other words, the grains can flow away from the rod without the aid of a slip shear band below the pestle. However, when the booming dune surface was impacted sharply by the palm of the hand, there was emission with $f_{d} \approx 73 \mathrm{~Hz}$ [5]. Evidently, the relatively large area of the impacting hand resulted in a sufficiently large degree of confinement that resulted in a slip shear band a few cm below the hand. Similarly, when booming grains were confined in a $25 \mathrm{~cm}$ wide circular channel and pushed by a large blade, they became boomable and or singable [9]. Additionally, they became singable when confined inside a jar [10]. In [1, 30], it is shown that salt, sugar and silent sand grains can exhibit singability when sufficiently confined. On the other hand, when a flat pile of the Brevort River sand was impacted vertically by the flat end of a wood rod (block) $14 \mathrm{~cm}$ in diameter, there was practically no musical sound emission, as was the case for the smaller block described above, due to the relatively low flowability of the grains.

\section{The pushed booming sand}

During the visit of the authors of [14] to the Sand Mountain, NEV, USA, the sand would not boom during free avalanche, but it would emit musical sounds when pushed by the hand or sheared (squeezed) by a shovel blade. Figure. 4 in [14] depicts the frequency spectra of the 
microphone and geophone recorded signals when a $30 \times 30 \mathrm{~cm}$ flat shovel blade was withdrawn sharply with a downward push from the dune face. There are distinct peaks at 67 and $76 \mathrm{~Hz}$ in the geophone spectrum, most likely due to distinct slip shear bands under the shovel. In the microphone spectrum, there are several peaks centered at around $60 \mathrm{~Hz}$ and a harmonic peak at about $120 \mathrm{~Hz}$, which is also present in Fig. $3_{d}$ in [14]. There is also a hint of a harmonic presence in Fig. $5_{b}$ and reference to possible low frequency content in the 3 to $30 \mathrm{~Hz}$ range, when the sand was dug by the hand.

The first attempt to determine the change in frequency with the manner the sand was pushed on the face of a dune can be found in [16]. It was determined that when the sand was pushed uphill, the frequency increased as opposed to when it was pushed downhill, and it also increased with the speed of push. In Fig. 2 in [28], the shear plane is depicted when a sand bed is pushed by a blade and an overburden is heaped up in front of the blade to a height considerably larger than the sand bed depth. The shear plane runs from the foot of the blade to the front of the overburden at an angle, $\beta$, from the horizontal bed plane. Intuitively, it could be argued that when the sand is pushed downhill, $\beta$ tends to zero, and that it tends to larger values when the sand is pushed uphill, resulting in lower overburden mass $M_{p}$, and in higher frequency $f_{d}=f_{p}$. It is possible, but unlikely that the sound is due to grain column vibrations and that the column number, $N$, is lower when the sand is pushed uphill. Similar arguments could be used to explain why $f_{d}$ increases with blade velocity on a horizontal sand bed.

In [9], it is claimed that frequencies, $f_{d}$, as low as $25 \mathrm{~Hz}$ were obtained by pushing the booming sand on the face of a dune. In the YouTube presentation by the authors of [9], it can be observed that when the sand on the face of a booming dune was squeezed between the two hands, a fairly low frequency sound was emitted. It is highly likely that a slip shear band was formed between the hands and that the pestle (overburden) mode of vibration was excited with frequency, $f_{d}=f_{p}$. It is unlikely that the slip shear band was thick enough so as to result in, $f_{d}=f_{1}$. There is no evidence of sand avalanche that would result in such a sound. Again, the question would be resolved when the frequency spectrum becomes available. During such sand squeezing and the subsequent dropping of the sand on the dune surface, the sand appears to respond in the same manner as the Brevort River singing sand except for the lower value of $f_{d}$ by about a factor of four. 
Furthermore, in [9], the study of the change of the dominant frequency, $f_{d}$, with blade speed and height of the grain mass in front of the blade was quantified. Booming grains from the Atlantic shores of Morocco were pushed by a $25 \mathrm{~cm}$ blade in a cylindrical container, $1 \mathrm{~m}$ in dimeter. From Fig. 2 in that report, it can be inferred that for fixed blade velocity, $V_{b}, f_{d}$ varies nearly as the inverse of $\sqrt{H_{b}}$, where $H_{b}$ is the sand height in front of the blade, implying that $f_{d}=f_{p}$. However, the increased sand height could result in thicker slip shear band and in $f_{d}=f_{1}$. The ambiguity would be resolved when the spectra of the emitted signals are available.

\section{Freely avalanching booming sand}

When a sand band, several cm thick, is in a state of avalanche, it is effectively confined by the plane of the static sand below and the thin band of about 20 relatively fast moving surface layers above $[2,9]$. It can now be argued that the grain layers near the bottom of the avalanche front experience the greatest stress level when they decelerate sharply and are overtaken by the layers above, and that a slip shear band could exist in that region. It is possible that the so called "roar sound" emitted when the sand is pushed downhill in a heaped-up manner, [16], is due to grain column vibrations in such a front slip shear band. If there were no harmonics of $f_{d}$, then, such a sound would be due to the overburden vibration with frequency $f_{d}=f_{p}$. However, the "hum" that follows the "roar" represents a steady state acoustic emission that is independent of an avalanche front. It can be maintained by continuously digging a hole where the avalanching sand is deposited [16]. It is reported in [2, 9] that the minimum thickness of an avalanche for booming to occur is about $2 \mathrm{~cm}$, implying grain number, $N \geq 100$, with $\bar{d}=0.18 \mathrm{~mm}$.

Figure 5 in [16] depicts the ripples (waves) on the dune surface when the sand was pushed downhill in a heaped-up manner. There are several avalanche fronts followed by flat plateaulike segments. The author describes the sound as, "being due to the hum accompanying the roar". In the context of this approach, the roar would be due to column vibrations in the front slip shear bands, while the hum would be due to similar vibrations in the surface of the flat segments, as described below. The column vibrations in the entire avalanche area would be synchronized by the surface modes of wave propagation excited by the avalanching grains, $[5-7]$. 
In a static sand dune, the macroscopic elastic moduli increase with depth, $\zeta$, due to the increased normal stress at the grain contact areas resulting in larger contact band radii. According to the Hertz-Mindlin contact theory, both phase velocities increase with depth as $\zeta^{1 / 6}$. This relation was verified by Bachrach et al. [15] for unconsolidated beach sand, despite velocity discrepancies discussed below. If the column propagation velocity, $V_{c}$, in the avalanching sand band, increases similarly with $\zeta$, then, according to (9), the contact band radius, $R_{b}$, also increases as $\zeta^{1 / 6}$. As in the case of the impacted grains, the ratio of the average grain diameter, $\bar{d}$, to the contact band thickness, $b$, and the ratio $\bar{d} / R_{b}(1)$, at the bottom of the avalanching band, were chosen equal to 1000 and 45 respectively.

With $N=48$, the ratio, $\bar{d} / R_{b}(49)=45(48)^{1 / 6}=45 \times 1.91=86$, at the top of the avalanching sand band. Similarly, the phase velocity in the top contact band would be, $c_{p}(49)=c_{p}(1) / 1.91$, implying that $c_{p}(49)=1.6 \mathrm{~m} / \mathrm{s}$ if $c_{p}(1)=3 \mathrm{~m} / \mathrm{s}$. Thus, with overburden mass, $M_{p}$, equal to the mass of 20 fast avalanching grains and an assumed grain size distribution in a given column, the eigenfrequencies were computed as follows: $f_{p}=49 \mathrm{~Hz}, f_{1}=114 \mathrm{~Hz}, f_{2}=221 \mathrm{~Hz}, f_{3}=359$ $\mathrm{Hz}$ etc. It was determined that the mode corresponding to $f_{1}$ is characterized by a node at about the center of the column. This is in agreement with the experimental data depicted Fig. $3_{d}$ and the discussion that follows on page 4 in [7], where the authors claim that the particle (grain) displacement, along the avalanche direction, has a node near the center of the avalanching band.

The frequency, $f_{p}$, corresponds to the surface (overburden) mode of vibration. In Fig. 2, parts (b) and (f) in [4], there appears to be evidence of such low frequency content. A minor envelope centered at $30 \mathrm{~Hz}$, as well as envelopes at $2 f_{d}$ and $3 f_{d}$, with $f_{d} \approx 100 \mathrm{~Hz}$, can be seen in the geophone frequency spectrum of the avalanche signals available at the website in [2]. Permission to this end was obtained from the author. A similar spectrum structure can be viewed in a YouTube presentation by the authors of [4] seen above. Furthermore, harmonics of $f_{d}=100 \mathrm{~Hz}$ can be seen in the spectrum of the sound emitted by an induced free avalanche available in the YouTube presentation by the authors of [9] with the title, The Song of Dunes.

For $N=96$ at the depth of $H=1.73 \mathrm{~cm}, c_{p}(1)$ would be $2 \times 3.0=6.0 \mathrm{~m} / \mathrm{s}$, and the column propagation velocity, $V_{c}$, would be about $2 \times 6.0=12.0 \mathrm{~m} / \mathrm{s}$. It is appreciably lower than the phase speed of $40 \mathrm{~m} / \mathrm{s}$ measured by generating a sinusoidal wave on the dune surface by the use of a loud speaker [2]. However, it could not be argued that the wave so generated was 
limited to a surface band of only $2 \mathrm{~cm}$ thick. Moreover, when the wavelength of the surface wave was measured by the use of floaters, [2], it could not correspond to a wave limited to a band of $2 \mathrm{~cm}$, since the floaters would have disrupted such a band. It was determined in [2] that the wavelength of such a wave was the same inside and outside the avalanching band, thus establishing that the wave was anchored in the sand bed well below the avalanching band. If the compression phase velocity were equal to $12 \mathrm{~m} / \mathrm{s}$ in the top layer of the static sand below the avalanching sand band, then, at $10 \mathrm{~cm}$ below, i.e., 556 layers below the top layer, it would be $12 \times 556^{1 / 6}=34 \mathrm{~m} / \mathrm{s}$, a number close to $40 \mathrm{~m} / \mathrm{s}$. It appears likely that there is a gradual jump in the propagation velocity from about $12 \mathrm{~m} / \mathrm{s}$ at the bottom of the avalanching band to $40 \mathrm{~m} / \mathrm{s}$ in the static sand below.

In the report by Bonneau et al. [5], the properties of the fundamental surface mode, that would synchronize the grain-grain collisions and also the grain column vibrations in the present context, are shown on page 8 in [5]. The mode propagates with phase velocity equal to $32 \mathrm{~m} / \mathrm{s}$ and has frequency and wavelength equal to $100 \mathrm{~Hz}$ and $32 \mathrm{~cm}$ respectively. From the plots of the particle displacement along the direction of flow, Fig. 3 in [5], it follows that the mode extends to the depth, $H \approx 32 \mathrm{~cm}$. Thus, it could be assumed that the mode would not be affected by the relatively low values of the elastic moduli of the contact bands in the avalanching band. Furthermore, from the expressions of the elastic moduli on page 4 in [5], it follows that the macroscopic elastic moduli approach zero as the depth, $\zeta \rightarrow 0$, and thus, the macroscopic compression and shear phase velocities and by extension the contact phase velocities, $c_{p}(j), c_{s}(j)$ could approach zero as $\zeta \rightarrow 0$.

The issue of the path of propagation of surface waves on a pile of quartz sand grains is addressed in [15]. Fermat's principle is invoked in order to argue that such waves tend to follow paths well below the surface where the propagation velocity is higher. In Fig. 6 in the same report, it is shown that the measured propagation velocity is less than half the one predicted by the Hertz-Mindlin theory. The authors suggest that such a discrepancy is due to the presence of clay and water in the sand which could lower the macroscopic elastic moduli significantly. The discrepancy was removed by effectively reducing the shear modulus of the grains by the factor of about 100, Fig. 7 in [15]. In the same Figure, it can be observed that when the distance between the shot and the receiver was about $10 \mathrm{~cm}$, the propagation velocity was just below $50 \mathrm{~m} / \mathrm{s}$. However, even for this low separation distance, the propaga- 
tion path would lie well below the depth of $2 \mathrm{~cm}$. Furthermore, the authors recognized the difficulty in measuring the propagation velocity near the surface of a sand pile.

In the context of this study, it could be argued that the relatively low velocity of $50 \mathrm{~m} / \mathrm{s}$ is partly due to the partial fluidization of the grain contact bands, and since such fluidization is bound to be higher in the avalanching band, where the grains are subjected to higher levels of sliding, the propagation velocity would be appreciably lower in the same band. It is worthy of note that the sand grains were not reported as musical, singing or booming in [15], and this leads to the conclusion that fluidization in the contact bands is not a sufficient condition for boomability. Thus, it has to be concluded that, as in the case of the impacted grains, both, grain contact fluidization and the stick-slip effect are required for grain boomability.

It might not be totally out of place to suggest that the elastic wave used to determine the propagation velocities, $c_{s}, c_{p}$, in a sand pile alters the surface physical state of the grains and in the process it affects the outcome of the measurement. That is, at appreciable depths, the grains are consolidated enough and there is negligible grain-grain sliding due to the presence of the elastic wave. However, near the surface there could be appreciable grain-grain sliding resulting in grain contact fluidization and in phase velocities well below those predicted by the Hertz-Mindlin theory. Thus, the measured phase velocity decreases with increased amplitude of the seismic wave between the shot and the receiver.

During a silent avalanche on a dune surface or in the initial stage of an avalanche on a boomable sand dune, a given grain, in one of the 20 or so fast avalanching layers, generates a quasi-periodic elastic wave train composed of the impact pulses upon collisions with the grains in the layer below. The average time between collisions, $T_{c}$, is given in the first paragraph in the Introduction. The chance that the given grain will collide with a grain below is maximum when the grain below collides with another grain in the layer further below, since then it is nearly stationary. Thus, it can be argued that the various quasi-periodic wave trains are somewhat synchronized and the resultant wave amplitude is sufficiently large to initiate the excitation of the column vibrations below. In this sense, the column vibrations, within a radius of several grain diameters, are nearly in phase, and if the conditions for the applicability of the stick-slip effect exist, the resultant amplitude of the column vibrations will be sufficient to generate the surface waves that synchronize all column vibrations within areas as large as several $\mathrm{m}^{2}$ as reported in [2]. Thus, the question arises as to whether a geophone buried a few 
cm below a silent avalanche can record a fairly monochromatic signal, as in the case of the impacted silent grains.

The collective grain column vibrations cannot be sustained at frequencies other than the average grain-grain collision rate, $f_{c} \approx 100 \mathrm{~Hz}$ for $\bar{d} \approx 0.18 \mathrm{~mm}$. Unlike the case of the singing grains where the energy that excites the column vibrations is derived from the impacting pestle, in this case the energy is derived mainly from the surface grain-grain collisions. Thus, any collective vibration has to be slaved to the frequency $f_{c}$. However, the frequency $f_{1}=f_{d}$ is defined by the elastic moduli of the contact shear bands, for a given $N$, and if $f_{d}$ is appreciably different from $f_{c}$, then, boomability is not possible. Such a conclusion is consistent with the rarity of such a phenomenon. It occurs only during certain periods of the year and not all dunes in a given region can boom during a free avalanche, even though, they can produce the booming sound when squeezed by a shovel blade [14] or pushed by the hand or a blade [9]. Furthermore, if the thickness of the avalanching sand band becomes too thin, then, $f_{d}$ is forced to exceed $f_{c}$ and boomability ceases, as was reported in $[5,9]$.

\section{Sliding booming sand plates}

Along the path of many suggestions and assumptions during the development of this study, not always correct, it seems fair to assume that there is a slip shear band (column shear band), in front of the sliding plate, responsible for the seismic and acoustic emissions. How far such a channel extends below the plate remains an open question. The channel width would be rather thin given the chaotic dynamics of the grain motion under the plate. Thus, the relatively low value of $f_{d}$ would imply the single spring mode of vibration where the relatively large overburden mass, $M_{p}$, would be responsible for the low value of $f_{d}$.

As in the case of the impacted grains, the gravitational energy of the plate can be transferred to the column vibrations via the stick-slip effect. At this stage, it is deemed appropriate to include an excerpt from the book by Curzon [31], p. 285, that appears to correspond to the observation by Vriend et al. [4] in that dune vibrations were detected even when there was no apparent avalanche in progress. "By the flowing in of the sand from the sides and the repeated tread [of the traveler] a large part of the whole sand-layer of the slope at last acquires motion, and by its friction against the motionless under-layer produces a noise, which from a humming becomes a murmur, and in the end passes into a roar, and is all the more surprising 
in that one sees but little of the trickling and general movement of the sand-layer."

\section{Conclusions}

The present theoretical approach (model) is based on the modes of vibration in grain columns, where the grain contact shear bands provide the compression and shear elastic strength and also the means for the transfer of energy from the impacting pestle, or from the potential energy of an avalanching sand mass, into such modes of vibration via the stick-slip effect. Such an approach is in agreement with the viewpoint, found in the literature, that the origin of such phenomena lies in the surface state of the grains producing the singing or booming sounds.

Within the context of this approach, it is possible to provide an explanation of the various questions raised in the Introduction, namely:

(a) The harmonics and the low frequency envelope observed in the spectra of the seismic and acoustic signals from a freely avalanching sand band correspond to column modes of vibration with frequencies equal to the harmonics of the fundamental, $f_{1}$, and the low frequency envelope at the frequency $f_{p}$ corresponds to the overburden mode of vibration, where the entire grain column acts as a single short spring.

(b) The same explanation holds regarding the observed frequency spectra of the signals from an impacted grain bed except that the low frequency envelope corresponds to the pestle mode of vibration.

(c) The singability of an impacted grain bed is the result of sufficient grain-grain slippage in the slip shear band, comprising the grain columns below the pestle, and of the applicability of the stick-slip effect. The pestle forces the grain columns to vibrate in phase with it, resulting in the collective vibration of all columns. The ensuing intense vibration below the pestle facilitates the slipping of the grain mass away from the pestle and nearly eliminates the surface noise generated by the intense grain-grain rubbing and the chaotic grain column vibrations adjacent to the pestle near the surface.

(d) Singing grains do not boom when forced to free-avalanche since their relatively low flowability does not allow for an orderly surface flow comprising a fast avalanching thin band and a slower well defined band below, several cm thick. Similarly, booming sand grains do not sing when impacted in a dish by a pestle since their relatively high flowability results in suffi- 
cient sand motion away from the pestle without the need for an intense vibration in the slip shear band below the pestle. It could be argued that ordinary sand grains do not sing when impacted in a dish since the stick-slip effect is not applicable in the grain contact areas, and this is likely the case during a silent sand avalanche.

(e) The relatively low propagation velocity of an elastic wave near the surface of a booming dune and more generally in a pile of ordinary sand, about $50 \mathrm{~m} / \mathrm{s}$ as opposed to about 200 $\mathrm{m} / \mathrm{s}$, could be attributed to the partial fluidization of the grain asperities inside the grain contact shear bands. In the same sense, when an elastic wave is used to determine its velocity of propagation, between the shot and the receiver, the unconsolidated grains near the surface would slide somewhat past one another and in the process the elastic wave affects the outcome of the measurement. That is, the measured propagation velocity would decrease with increased wave amplitude.

(f) The extreme sensitivity of the boomability of a given sand dune to weather and other factors could be attributed to its sensitive dependence on the value of the fundamental frequency $f_{1}$ of the column vibrations in the avalanching band. That is, if $f_{1} \neq f_{c}$, boomability cannot occur, where, $f_{c}=1 / T_{c}$ and $T_{c}$ is the average time required for one grain to overtake another

in the fast avalanching surface band of about 20 layers. Furthermore, if the avalanching band is too thin, then, $f_{1}$ becomes greater than $f_{c}$ and boomability ceases. Additionally, boomability would not be possible if the stick-slip effect were not applicable.

(g) Whereas, it is highly unlikely that the dominant frequency, $f_{d}$, is equal to the low pestle frequency, $f_{p}$, when a grain bed is impacted by a pestle, this is not the case when booming sand grains are impacted (sheared, pushed) by a large blade, or when sand plates break off on the face of a dune and slide downhill. In the latter cases, the maintenance of a slip shear band with thickness equal to about $20 \mathrm{~mm}$, as in the case of a free avalanche, is highly unlikely due to the relatively violent grain motion in front of the blade. It is more likely that the slip shear band is only a few mm thick and that $f_{d}=f_{p}$.

\section{Acknowledgment}

Considerations are due to the Research, Development and Creativity Office of Laurentian University for their financial support, and to Garry Clark for his preparation of the diagrams and assistance on internet related questions. 


\section{References}

1. A. J. Patitsas. J. Natural and Physical Sciences. Vol. 2, Issue 1, (2008). Available from http/www.scientificjournals.org/journals2008/articles/1404.pdf.

2. B. Andreotti. Phys. Rev. Lett. 93, 238001 (2004).

3. GDR MiDi. Eur. Phys. J. E. 14, 341 (2004).

4. N. M. Vriend, M. L. Hunt, R. W. Clayton, C. E. Brennen, K. S. Brantley, and A. Ruiz-Angulo. Geophys. Res. Lett. 34, L16306 (2007).

5. L. Bonneau, B. Andreotti, and E. Clement. Phys. Rev. E. 75, 016602 (2007).

6. L. Bonneau, B. Andreotti, and E. Clement. Phys. Rev. Lett. 101, 118001 (2008).

7. B. Andreotti, and L. Bonneau. Phys. Rev. Lett. 103, 238001 (2009).

8. R. A. Bagnold. The Physics of Blown Sand and Desert Dunes, 247-257. Chapman and Hall, London, UK. 1941.

9. S. Douady, A. Manning, P. Hersen, H. Elbelrhiti, S. Protiere, A. Daerr, and B. Kabbachi. (2006). Phys. Rev. Lett. 97, 018002 (2006).

10. M. F. Leach, and A. G. Rubin. (1990). Progress in Acoustic Emission V, Proceedings of the 10th International Acoustic Emission Symposium, The Japanese Society for Non-destructive Inspection, Japan, 239 (1990).

11. F. Nori, P. Sholtz, and M. Bretz. (1997). Scientific American. 277, 84 (1997).

12. M. F. Leach, D. E. Goldsack, C. Kilkenny, and C. Filion. Proceedings of the 14th International Acoustic Emission Symposium and 5th Acoustic Emission World Meeting, Big Island, Hawaii, III 89 (1998).

13. B. Andreotti, L. Bonneau, and E. Clement. Geophys. Res. Lett. 35, L08306 (2008).

14. D. R. Criswell, J. F. Lindsay, and D. L. Reasoner. Journal of Geophysical Research. 80, 4963 (1975).

15. R. Bachrach, J. Dvorkin, and A. Nur. Geophysics. 63, No 4, 1234 (1998).

16. A. W. Lewis. South African Geographical Journal. 19, 33 (1936).

17. B. Bhushan. Principles and Applications of Tribology. John Wiley and Sons, New York, USA. 1999.

18. S. Miwa, J. Hidaka, and A. Shimosaka. Powder Science and Technology in Japan, 
KONA, 1, 64 (1983).

19. T. Brunet, X. Jia and P. Mills. Phys. Rev. Lett. 101, 138001 (2008).

20. A. J. Patitsas. Can. J. Phys. 88, 863 (2010).

21. P. K. Haff. American Scientist. 74, 376 (1986).

22. K. R. Symon. Mechanics, 3rd edition. Addison-Wesley, Reading, MA, USA, 1971.

23. H. F. Winterkorn, and H. Y. Fang. Foundation Engineering Handbook. Van Nostrand Reinhold Co., New York, USA, 1975.

24. K. Nishiyama, and S. Mori. Japanese J. of Appl. Phys. 21, No 4, 591 (1982).

25. A. W. Brown, W. A. Campbell, J. M. Jones, and E. R. Thomas. Proc. Univ. Newcastle Upon Tyne Philos. Soc. 1, 1 (1964).

26. H. Takahara. J. Acoust. Soc. Am. 53, No 2, 634 (1973).

27. P. Sholtz, M. Bretz, and F. Nori. Contemporary Physics. 38, No 5, 329 (1997).

28. R. A. Bagnold. Proc. Royal Soc. 295, 219 (1966).

29. D. W. Humphries. Sedimentology, 6, 135 (1966).

30. A. J. Patitsas. J. Fluids and Structures. 17, 287 (2003).

31. M. Curzon. Tales of Travel. Hodder and Stoughton, London, UK. 1923. 